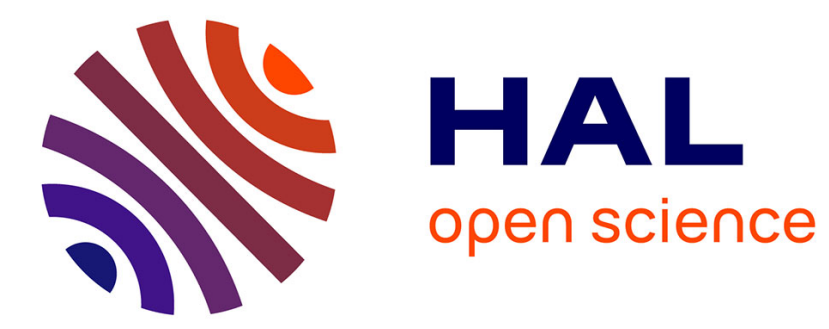

\title{
Analysis of Approval Voting in Poisson Games
}

François Durand, Antonin Macé, Matias Nunez

\section{To cite this version:}

François Durand, Antonin Macé, Matias Nunez. Analysis of Approval Voting in Poisson Games. 2019. halshs-02049865

\section{HAL Id: halshs-02049865 https://shs.hal.science/halshs-02049865}

Preprint submitted on 26 Feb 2019

HAL is a multi-disciplinary open access archive for the deposit and dissemination of scientific research documents, whether they are published or not. The documents may come from teaching and research institutions in France or abroad, or from public or private research centers.
L'archive ouverte pluridisciplinaire HAL, est destinée au dépôt et à la diffusion de documents scientifiques de niveau recherche, publiés ou non, émanant des établissements d'enseignement et de recherche français ou étrangers, des laboratoires publics ou privés. 


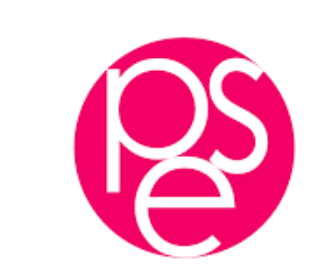

PARISSCHOOL OFECONOMICS
ECOLED'ECONOMIEDE PARIS

\title{
WORKING PAPER N № 2019 - 11
}

Analysis of Approval Voting in Poisson Games

\author{
François Durand \\ Antonin Macé \\ Matias Nunez
}

JEL Codes: D72; C72; C63

Keywords : Approval voting, Poisson games, Stable equilibria, Monte-Carlo simulations.
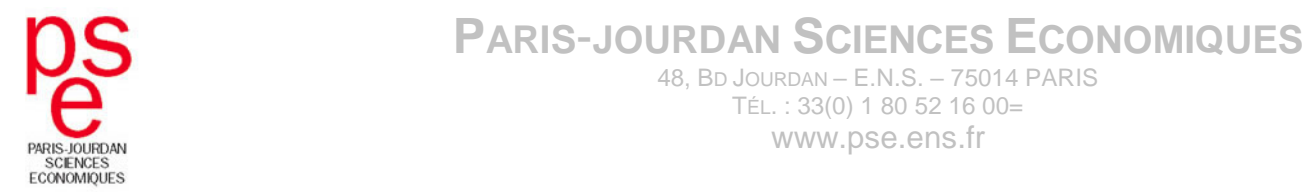


\title{
ANALYSIS OF APPROVAL VOTING IN POISSON GAMES*
}

\author{
FRANCOIS DURAND ${ }^{\mathrm{a}}$, ANTONIN MACÉb ${ }^{\text {, AND MATÍAS NÚÑEZ }}$
}

\begin{abstract}
We analyze Approval Voting in Poisson games endowing voters with private values over three candidates. We first show that any stable equilibrium is discriminatory: one candidate is commonly regarded as out of contention. We fully characterize stable equilibria and divide them into two classes. In direct equilibria, best responses depend only on ordinal preferences. In indirect equilibria, preference intensities matter. Counter-intuitively, any stable equilibrium violates the ordering conditions, a set of belief restrictions used to derive early results in the literature. We finally use Monte-Carlo simulations to estimate the prevalence of the different sorts of equilibria and their likelihood to elect a Condorcet winner.
\end{abstract}

KEYworDs. Approval voting, Poisson games, Stable equilibria, Monte-Carlo simulations.

JEL Classification. D72; C72; C63.

How does the electoral outcome depend on the voting rule? This question, quite vast, has been addressed by both economists and political scientists in order to set the basis of electoral engineering. Particularly useful to answer this question are the pivotal voting models in which voters rationally choose the ballot that maximizes their expected utility. In these models, the prediction tool is the equilibrium concept: each voter casts the ballot that maximizes his expected utility given his beliefs concerning the types and the actions of the rest of voters. Among the different findings, a key one concerns first-past-the-post voting methods, where voters vote for one candidate. Any such rule generates several Duvergerian (or discriminatory) equilibria in which all votes are concentrated in two candidates (see Myerson and Weber [1993], Myerson [2002], Goertz and Maniquet [2011] and Bouton and Gratton [2015] among others). This multiplicity of equilibria is particularly unappealing since they may be disconnected from the preferences of the electorate: as long as voters anticipate that voting is almost surely pivotal between some pair of candidates, the votes become concentrated on these two candidates which in turn validates the voters' anticipations.

It has been advocated [Brams, 1980, Myerson, 2002, Laslier, 2009, Goertz and Maniquet, 2011, Bouton and Castanheira, 2012, Ahn and Oliveros, 2016] that Approval Voting (henceforth AV) might prevent the previously mentioned flaws of first-past-the-post voting methods. Under this rule, a voter can vote for as many candidates as he wants, and the one(s) with the most votes wins the election. The main advantage of $\mathrm{AV}$ is that it gives no constraints to the voters regarding the number of approved candidates and this can lead to huge gains from

\footnotetext{
* We would like to thank Laurent Bouton, Tasos Kalandrakis, Yukio Koriyama, Jérôme Lang, Klaus Nehring and Yulliy Sannikov for useful comments. Financial support from the ANR-14-CE24-0007-01 (CoCoRICoCoDEC) project and the ANR-17-EURE-001 (a French government subsidy managed by the Agence Nationale de la Recherche under the framework of the Investissements d'avenir programme) is acknowledged.

a NOKIA-BeLl LABS FRANCE. This author carried out the work presented in this paper at LINCS (www. lincs. $\mathrm{fr}$ ).

b PARIS SCHOOL OF ECONOMICS, CNRS AND ENS, FRANCE.

c Université Paris-Dauphine, PSL Research University, CNRS, LAmsade, 75016 Paris, France.

Date: February 26, 2019.
} 
a collective perspective. Laslier [2009] and Myerson and Weber [1993] deliver a very sharp prediction: namely, in equilibrium, this rule tends to uniquely select the Condorcet winner if it exists. This prediction holds in the model of Myerson and Weber [1993], where voters' beliefs meet the following two properties: (i) the higher the perceived score of a candidate, the infinitely more likely that a vote for him becomes pivotal and (ii) pivot events involving two candidates are infinitely more likely than pivot events in which three or more candidates are involved. We refer in the sequel to these two properties as the ordering conditions. Building on it, voters' best responses are immediate since candidates' perceived scores are enough to determine the optimal ballots (see Laslier [2009] for a precise statement). Figure 1 illustrates these best responses with three candidates ${ }^{1}$. Since $a$ and $b$ are perceived as the most serious contenders, voters who prefer $a$ to $b$ approve $a$ and those who prefer $b$ to $a$ vote for $b$. Approving of $c$ is a best response if and only if $c$ is preferred to the perceived winner, candidate $a$. With this sharp description of best responses, the logic of the unique equilibrium is rather clear: only a Condorcet Winner can be elected.

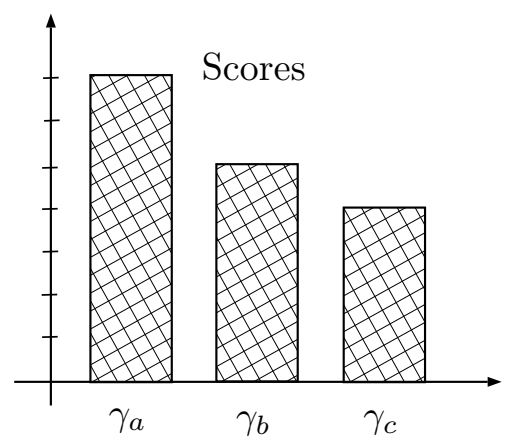

Ordering conditions

$\mathbb{P}\left[\right.$ piv $\left._{a b}\right] \gg \mathbb{P}\left[\right.$ piv $\left._{a c}\right] \gg \mathbb{P}\left[\right.$ piv $\left._{b c}\right] \gg \mathbb{P}\left[\operatorname{tie}_{a b c}\right]$

Best responses

1. If $a>b$, approve $a$ (and possibly $c$ )

2. If $b>a$, approve $b$ (and possibly $c$ )

3. Approve $c$ iff $c>a$

FiguRE 1. Ordering Conditions.

Yet, the status of AV in Myerson's Poisson games is rather uncertain. Poisson games are used for several applications in mechanism design and, in particular, they have become the classical model to understand large elections from a game-theoretical perspective. In these games, the size of the electorate follows a Poisson distribution, and the strategic decision of each voter is based on endogenous pivot probabilities, rather than on ad-hoc belief restrictions. Myerson [2002] presents an elegant example that shows a nice feature of AV in this model: when all voters unanimously prefer some candidate, AV uniquely selects the Condorcet winner in equilibrium whereas the Plurality rule fails to do so. However, as proved by Núñez [2010], one can find examples in which $\mathrm{AV}$ fails to induce the victory of a candidate ranked first by a majority of the electorate. Beyond these specific examples, little is known on AV equilibria in general $^{2}$, and the comparison to first-past-the-post voting methods seems difficult to establish.

Our work is the first one to give a general description of the equilibria in this game. As a starting point, we present an in-depth description of the voter's decision problem in order to identify the precise events that matter to determine best responses. Focusing on stable equilibria, our first main contribution is to show that these equilibria must be discriminatory. In such an equilibrium, a pair of candidates (say $a$ and $b$ ) acts as a coordinating device: each voter

\footnotetext{
${ }^{1}$ We focus exclusively on a setting with three candidates as the vast majority of works in Poisson games.

${ }^{2}$ Myerson [2002] only provides general results for bipolar elections, in which the electorate can be divided in two antagonist sides.
} 
anticipates that, conditional on being pivotal, his vote will almost surely determine whether $a$ or $b$ wins the election. Thus, each voter approves either $a$ or $b$ but no voter approves both or disapproves both. ${ }^{3}$

In contrast with first-past-the-post voting, the previous observation is not sufficient to determine best responses, as each voter may choose to approve $c$ or not. This choice is driven by the relative probabilities of the other pivots (being decisive between $a$ and $c$, or between $b$ and $c$ ). In a first class of direct equilibria, the ratio of these probabilities diverge, and best responses are straightforward. However, these equilibria often fail to exist. We then characterize the remaining class of indirect equilibria, exhibiting finite pivot probability ratios, and for which best responses may depend on cardinal utilities. We show that, for every ordinal preference profile, there is an open set of cardinal representations of these preferences for which such an indirect equilibrium exists. Moreover, if a Condorcet winner exists, the constructed indirect equilibrium elects him.

As a by-product of our analysis, we obtain an important and striking result: the ordering conditions are never satisfied in a stable equilibrium (both conditions (i) and (ii) are violated). This finding underscores the discrepancy between the Myerson-Weber model and the model of Poisson games. Finally, we exploit our characterization of stable equilibria to analyze numerically the prevalence of direct and indirect equilibria, and to assess the likelihood of electing a Condorcet winner when it exists.

\section{Approval Voting Games}

\subsection{Candidates and voters}

We follow the model stated by Myerson [2002] and thus consider an election in which voters elect one candidate into office from a set of candidates $\mathscr{K}=\{a, b, c\}$. The number of voters is drawn from a Poisson distribution with mean $n .^{4}$ It follows that the common knowledge probability that exactly $p$ voters take part in the election equals $e^{-n} \frac{n^{p}}{p !}$.

A voter's type $t$ determines its cardinal preferences. Throughout, we assume that preferences are strict and that two voters with the same ordinal preferences share the same cardinal preferences. For any preference order over the candidates $i>j>k$, the corresponding type is denoted by $t:=i j$. Up to renormalization, we assume that voters of this type have utility 1 (resp. 0) for the victory of candidates $i$ (resp. $k$ ), and we denote by $u_{t} \in(0,1)$ their utility for the victory of candidate $j$. The type space is denoted by $\mathscr{T}=\{a b, a c, b a, b c, c a, c b\}$. Each voter's type is independently drawn from $\mathscr{T}$ according to the preference profile denoted by $r=\left(r_{t}\right)_{t \in \mathscr{T}}$ with $\sum_{t \in \mathscr{T}} r_{t}=1$. We assume that for any subsets of types $T, T^{\prime} \subseteq \mathscr{T}$, with $T \neq T^{\prime}$, $\sum_{t \in T} r_{t} \neq \sum_{t \in T^{\prime}} r_{t}$. Note that this implies that $r_{t}>0$ for each $t \in \mathscr{T}$. This assumption is imposed to simplify the statements of the different results, yet it can be relaxed at the cost of more cumbersome notation and is indeed relaxed in most examples for the purpose of simplicity. To

\footnotetext{
${ }^{3}$ The existence of discriminatory equilibria is not unattractive per se: under first-past-the-post, the problem lies in their multiplicity [Myerson, 2002].

${ }^{4}$ In these games [Myerson, 1998], the voters' actions uniquely depend on their private information $t$ (common public information). Moreover, the number of voters who choose a given ballot is independent of the number of voters who choose another ballot (independence of actions). These two properties are used throughout.
} 
assess the relevance of some results, we sometimes refer to a measure on the set of preference profiles $r$, which is computed with the uniform distribution on the simplex over $\mathscr{T}$.

We focus on elections held under AV: voters cast a ballot for as many candidates as they want, and the candidate receiving the highest number of votes is elected (ties are broken with equal probability). Each voter simultaneously selects one message chosen in the set of approval voting ballots $\mathscr{M}:=\{a, b, c, a b, a c, b c\}$. For instance, $a$ stands for the ballot that approves candidate $a$ whereas $b c$ is the one that approves both $b$ and $c$. For any ballot $m$ and candidate $k$, we note $k \in m$ if $k$ is approved in $m$.

\subsection{Strategies and Equilibria.}

As usual in these models, strategies are defined type by type so that all agents of the same type play the same strategy. Namely, a pure strategy profile is a vector $\sigma=\left(\sigma_{t}\right)_{t \in \mathscr{T}}$, where $\sigma_{t} \in \mathscr{M}$ for each type $t$. For example, if $\sigma_{a b}=c$, then voters of type $a b$ choose the ballot $c$. Then, for any ballot $m \in \mathscr{M}$ and any candidate $k \in \mathscr{K}$, we write

$$
\begin{array}{ccccc}
\text { Share of ballot } m: & Z_{m} \sim \mathscr{P}\left(n \tau_{m}\right) \quad \text { with } \quad \text { expected share } & \tau_{m}=\sum_{t \mid \sigma_{t}=m} r_{t}, \\
\text { Score of candidate } k: & S_{k} \sim \mathscr{P}\left(n \gamma_{k}\right) \quad \text { with } \quad \text { expected score } \quad \gamma_{k}=\sum_{m \mid k \in m} \tau_{m} .
\end{array}
$$

Note that the ballot profile $\tau=\left(\tau_{m}\right)_{m \in \mathscr{M}}$ meets $\sum_{m \in \mathscr{M}} \tau_{m}=1$. The ballot shares are independent random variables whereas the candidate scores may be correlated since each voter can approve of several candidates. We say that a candidate $i$ is elected at a strategy profile $\sigma$ if $\gamma_{i}>\gamma_{j}, \gamma_{k}$, as this implies that the score of $i$ is above those of both $j$ and $k$ with a probability approaching to one when $n$ gets large.

The set $Z \subseteq \mathbb{N}^{\mathcal{M}}$ describes the possible ballot outcomes of the election, a typical element is a vector $z=\left(z_{m}\right)_{m \in \mathscr{M}}$ where each component $z_{m}$ denotes the number of voters choosing ballot $m$. An event is a subset of ballot outcomes $E \subseteq \mathcal{Z}$. Slightly abusing notation, we write $z+m$ for the vector $z+1_{m}$ (when we add 1 to the $m$-component only) and $E-m$ for the event $\left\{z \in \mathcal{Z} \mid z+1_{m} \in E\right\}$. The probability of a ballot outcome $z \in \mathcal{Z}$ is then given by:

$$
\mathbb{P}[z \mid n \tau]=\prod_{m \in \mathscr{M}}\left(\frac{e^{-n \tau_{m}}\left(n \tau_{m}\right)^{z_{m}}}{z_{m} !}\right) .
$$

For each ballot outcome $z \in \mathcal{Z}$ and each candidate $k$, the score of $k$ at $z$ is noted $s_{k}(z)$ and the set $K(z)$ stands for the set of candidates with the maximal score at $z$ :

$$
s_{k}(z)=\sum_{m \mid k \in m} z_{m} \text { and } K(z)=\underset{k \in \mathcal{K}}{\arg \max } s_{k}(z) .
$$

Since ties are broken by a fair coin toss, the probability of a candidate winning the election at $z$ equals $\frac{1}{\# K(z)}$ for each candidate with a maximal score. Building on the previous notations, the expected utility for a voter of type $t$ when voting $m$ is denoted by

$$
U_{t}[m \mid n \tau]=\sum_{z \in \mathscr{Z}} U_{t}[m \mid z] \mathbb{P}[z \mid n \tau],
$$

where $U_{t}[m \mid z]$ denotes the average utility of candidates in $K(z+m)$ for type $t$. 
Therefore, the best response to a strategy profile $\sigma$ depends only on $n \tau$. It can be written as $\mathrm{BR}(\cdot \mid n \tau) \subseteq \mathscr{M}^{\mathscr{T}}$, where, $\forall t \in \mathscr{T}, \mathrm{BR}(t \mid n \tau)=\arg _{\max } \in \mathscr{M}_{t}[m \mid n \tau]$. We focus on a large electorate, that is, we consider the limit behaviour when its expected size $n$ tends to infinity.

Voting equilibria are sometimes criticized in the literature for hinging on too precise beliefs held by voters [Fey, 1997]. In particular, Poisson voting models with private values exhibit an absence of aggregate uncertainty, so that equilibria may rest on the knowledge of the precise ballot profile $\tau$. To address this concern, we focus on stable equilibria, which are immune to the introduction of a small dose of aggregate uncertainty. Formally, this refinement coincides with the concept of strictly perfect equilibrium for normal-form games [Okada, 1981], already employed in Poisson games by Bouton and Gratton [2015].

Definition 1. A strategy profile $\sigma^{*}=\left(\sigma_{t}^{*}\right)_{t \in \mathscr{T}}$ is a stable equilibrium if $\exists \varepsilon>0$ and $n_{0} \geq 0$ such that for any ballot profile $\tau$ in the simplex over $\mathscr{M}$ with $\left|\tau-\tau^{*}\right|<\varepsilon$ and $n \geq n_{0}$, we have $\sigma_{t}^{*} \in \mathrm{BR}(t \mid n \tau)$, where $\tau^{*}$ denotes the ballot profile associated to $\sigma^{*}$.

\subsection{Best Responses}

For any voter with type $t$ and $i \in\{1,2,3\}$, we denote by $t_{i}$ the candidate in $i$-th position in his preference ranking (for example, $t_{1}$ is his most liked candidate). By a slight abuse of notation, $t_{1}$ denotes a ballot in favor of his best candidate and $t_{1} t_{2}$ a ballot in favor of his two best candidates. Thus, depending on the context, $a$ may denote the ballot $a$ or the candidate $a$, and $a b$ may denote the ballot $a b$ or the type $a b$. It can be shown that, ${ }^{5}$ for each ballot profile $\tau$, each $n$ and each type $t, U_{t}\left[t_{1} \mid n \tau\right], U_{t}\left[t_{1} t_{2} \mid n \tau\right]>U_{t}[m \mid n \tau]$ for any $m \in \mathscr{M} \backslash\left\{t_{1}, t_{1} t_{2}\right\}$. It follows that w.l.o.g. the strategy set of each type $t$ can be restricted from $\mathscr{M}$ to $\mathscr{M}^{t}=\left\{t_{1}, t_{1} t_{2}\right\}$, simplifying the strategic analysis.

Building on this observation, we introduce a graphical representation of the voting game we analyze throughout. We represent on Figure 2 the Approval game for an arbitrary preference profile $r$. Each type of voter $t$ is located between the half-lines pointing to the ballots $t_{1}$ and $t_{1} t_{2}$. For instance, a voter of type $a b$ chooses between ballot $a$ (to her left) and ballot $a b$ (to her right).

For each size of the electorate $n$ and each ballot profile $\tau$, the decision of a voter of type $t$ reduces to determining the sign of the following expression:

$$
\Delta(t \mid n \tau)=U_{t}\left[t_{1} \mid n \tau\right]-U_{t}\left[t_{1} t_{2} \mid n \tau\right]=\sum_{z \in Z} \mathbb{P}[z \mid n \tau]\left(U_{t}\left[t_{1} \mid z\right]-U_{t}\left[t_{1} t_{2} \mid z\right]\right) .
$$

As is well-known, for most election outcomes, altering a single ballot does not affect the winner of the election. It follows that relatively few outcomes determine voters' best responses. Expression (1) can be greatly simplified by focusing only on pivot and tie events, for which the winner of the election can indeed be affected by a single ballot, which we define below.

\footnotetext{
${ }_{5}$ More precisely, in a framework with a fixed number of voters, it is weakly dominant for a voter to approve of his best candidate and it is weakly dominated to vote for his worst candidate (see De Sinopoli et al. [2006], Laslier and Sanver [2010] and Bouton and Castanheira [2012]). For any $t \in \mathscr{T}$ and any $z \in \mathcal{Z}, U_{t}\left[t_{1} \mid z\right], U_{t}\left[t_{1} t_{2} \mid z\right] \geq U_{t}[m \mid$ $z]$ for any $m \in \mathscr{M} \backslash\left\{t_{1}, t_{1} t_{2}\right\}$. Moreover, for any size $n$ of the electorate and any ballot profile $\tau$, the event in which there is exactly one voter occurs with positive probability. In this event, the previously mentioned dominance relations become strict since each additional point matters to determine the winner.
} 


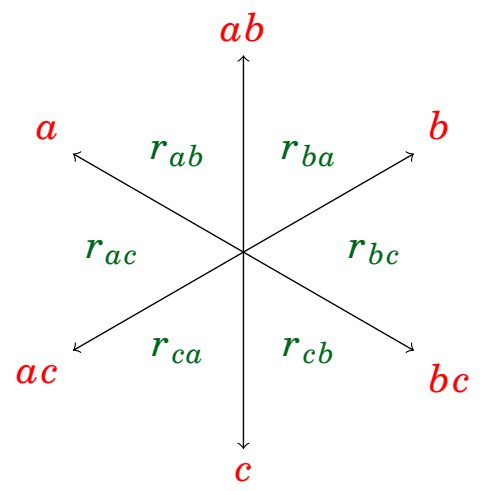

FigURE 2. Graphical representation of the Approval game.

For each pair $i j$ of candidates (and with $k$ denoting the remaining candidate), we write

$$
\operatorname{piv}_{i j}=\left\{z \in \mathcal{Z} \mid s_{i}(z)=s_{j}(z)>s_{k}(z)\right\},
$$

the pivot event where candidates $i$ and $j$ are tied for victory. For each $K \subseteq \mathscr{K}$, we let

$$
\operatorname{tie}_{K}=\left\{z \in \mathcal{Z} \mid s_{i}(z)=s_{j}(z), \forall i, j \in K\right\}
$$

stand for the event where all candidates in $K$ are tied.

For each pair $i j$ of candidates and each type $t \in \mathscr{T}$, we denote by $\operatorname{piv}_{i j}^{t}$ the event where casting a ballot $t_{1}$ or $t_{1} t_{2}$ can create a pivot between candidates $i$ and $j$ so that

$$
\operatorname{piv}_{i j}^{t}=\left(\operatorname{piv}_{i j}-t_{1}\right) \cup\left(\operatorname{piv}_{i j}-t_{1} t_{2}\right) .
$$

For each type $t \in \mathscr{T}$, we denote:

$$
\operatorname{tie}_{a b c}^{t, 1}=\operatorname{tie}_{a b c}-t_{1} \text { and } \operatorname{tie}_{a b c}^{t, 2}=\operatorname{tie}_{a b c}-t_{1} t_{2},
$$

the events where casting a ballot $t_{1}$ or $t_{1} t_{2}$ (respectively) can create a tie between the three candidates.

Equipped with the previous notations, the following proposition shows how the utility difference $\Delta(t \mid n \tau)$ can be expressed as a function of pivot and tie probabilities.

Proposition 1. For each ballot profile $\tau$ and each size $n$ of the electorate, the utility difference $\Delta(t \mid n \tau)$ for a type $t$ can be expressed as:

$$
\mathbb{P}\left[\operatorname{piv}_{t_{1} t_{2}}^{t} \mid n \tau\right]\left(\frac{1-u_{t}}{2}\right)+\mathbb{P}\left[\operatorname{piv}_{t_{2} t_{3}}^{t} \mid n \tau\right]\left(\frac{-u_{t}}{2}\right)+\left(2 \mathbb{P}\left[\operatorname{tie}_{a b c}^{t, 1} \mid n \tau\right]+\mathbb{P}\left[\operatorname{tie}_{a b c}^{t, 2} \mid n \tau\right]\right)\left(\frac{1-2 u_{t}}{6}\right) .
$$

The main lesson of the proposition is that the best response of a voter in this game simply depends on the sign of $\Delta$, which is a decreasing function of $u_{t}$. Therefore, when $n$ is large enough, for each ballot profile $\tau$ and each type $t$, there is some value $u_{t}^{*} \in[0,1]$ such that the best responses are given by:

$$
\operatorname{BR}(t \mid n \tau)=\left\{\begin{array}{llll}
t_{1} & \text { if } \Delta(t \mid n \tau)>0 & \Longleftrightarrow u_{t}<u_{t}^{*} \\
t_{1} t_{2} & \text { if } \Delta(t \mid n \tau)<0 & \Longleftrightarrow u_{t}>u_{t}^{*} .
\end{array}\right.
$$

The precise value of $u_{t}^{*}$ depends on the ballot profile $\tau$ and depends on the relative likelihood of pivot and tie events. For instance, consider a voter with type $t$ who believes that piv $t_{2} t_{3}$, 
$\operatorname{tie}_{a b c}^{t, 1}$ and tie ${ }_{a b c}^{t, 2}$ are negligible with respect to the pivot event $\operatorname{piv}_{t_{1} t_{2}}^{t}$. His decision is then clear since

$$
\Delta(t \mid n \tau) \sim_{n \rightarrow \infty} \mathbb{P}\left[\operatorname{piv}_{t_{1} t_{2}}^{t} \mid n \tau\right]\left(\frac{1-u_{t}}{2}\right) \quad \Longrightarrow \quad \Delta(t \mid n \tau)>0 \text { for each } u_{t} \in(0,1) .
$$

In this case, $\operatorname{BR}(t \mid n \tau)=t_{1}$ and $u_{t}^{*}=1$. A symmetric argument applies if he believes that $\operatorname{piv}_{t_{2}}^{t} t_{3}$ is the only serious pivot event, implying that $\mathrm{BR}(t \mid n \tau)=t_{1} t_{2}$ and $u_{t}^{*}=0$.

Yet, it may also happen that a voter believes that $\operatorname{piv}_{t_{1} t_{2}}^{t}, \operatorname{piv}_{t_{2} t_{3}}^{t}, \operatorname{tie}_{a b c}^{t, 1}$ and tie ${ }_{a b c}^{t, 2}$ exhibit finite pairwise probability ratios. In that case (and only in that case), these ratios deliver a cutoff $u_{t}^{*} \in(0,1)$, such that the voter's best response is to vote $t_{1}$ if $u_{t}<u_{t}^{*}$, and to vote $t_{1} t_{2}$ if $u_{t}>u_{t}^{*}$. We provide an example of such a situation in Section 5.4.

\section{Pivot Probabilities ANd their Magnitudes}

It is well known that pivot probabilities are typically hard to compute explicitly. Early papers in the literature rely on combinatorial arguments (Palfrey and Rosenthal [1983, 1985]) or avoid any computation by referring to the ordering conditions (Myerson and Weber [1993]). A great insight from Myerson [2000] is to introduce the idea of a magnitude, which measures the rate of convergence of the probability of an event to zero: when two events have different magnitudes, their relative probabilities diverge.

Definition 2. The magnitude of an event $E \subseteq \mathcal{Z}$ is defined by $\mu(E)=\lim _{n \rightarrow \infty} \frac{\log (\mathbb{P}[E \mid n \tau])}{n}$.

With this definition, if $\mu(E)>\mu(F)$, then $\lim _{n \rightarrow \infty} \mathbb{P}[F \mid n \tau] / \mathbb{P}[E \mid n \tau]=0$ (but the converse is not true in general). The important advantage of working with magnitudes of pivot and tie events is that they can be computed explicitly as the solution of a constrained minimization program, thanks to the Dual Magnitude Theorem in Myerson [2002]. Building on this result, we characterize the values that relevant magnitudes can take.

Theorem 1. For any ballot profile $\tau$, any type $t$ and any pair $i j$ of candidates (denoting by $k$ the remaining candidate),

$$
\mu\left[\operatorname{piv}_{i j}^{t}\right]=\left\{\begin{array}{ll}
\mu\left[\mathrm{tie}_{i j}\right] & \text { if } \delta_{i j}(\tau)>0, \\
\mu\left[\mathrm{tie}_{a b c}\right] & \text { if } \delta_{i j}(\tau) \leq 0,
\end{array} \text { with } \delta_{i j}(\tau)=\tau_{i} \sqrt{\frac{\tau_{j}+\tau_{j k}}{\tau_{i}+\tau_{i k}}}+\tau_{i j}-\tau_{j k} \sqrt{\frac{\tau_{i}+\tau_{i k}}{\tau_{j}+\tau_{j k}}}-\tau_{k},\right.
$$

and

$$
\mu\left[\operatorname{tie}_{a b c}^{t, 1}\right]=\mu\left[\operatorname{tie}_{a b c}^{t, 2}\right]=\mu\left[\operatorname{tie}_{a b c}\right] .
$$

The theorem establishes two important properties: (i) the magnitudes of pivot events do not depend on types; and (ii) the magnitude of a pivot $i j$ equals either the magnitude of tie ${ }_{i j}$ or that of tie ${ }_{a b c}$. The intuition for the second point stems from the interpretation of the threshold $\delta_{i j}(\tau)$ as the difference between the expected score of $i$ and that of $k$, conditionally on tie ${ }_{i j}{ }^{6}$

\footnotetext{
${ }^{6}$ To see why, recall that Myerson [2000, 2002] apply large deviations techniques to show that the probability of a cone event $E$ (such as tie $i_{j}$ ) is concentrated (for large electorates) in outcomes for which the proportion of either ballot is (approximatively) fixed. For each ballot $m$, this proportion can be expressed as the product of the ballot share $\tau_{m}$ to its offset ratio $\phi_{m}$. The offset ratios can be obtained as the solutions of a constrained minimization program, whose value corresponds to the magnitude of the event $E$ (this is the Dual Magnitude Theorem in Myerson [2002]). For $E=\operatorname{tie}_{i j}$, it is easy to see that $\phi_{k}=\phi_{i j}=1$ (ballots $i j$ and $k$ do not influence the relative scores of $i$ and $j$ ), while $\phi_{i}=1 / \phi_{j k}=\sqrt{\left(\tau_{j}+\tau_{j k}\right) /\left(\tau_{i}+\tau_{i k}\right)}$. Therefore, the most likely difference in score between $i$ and $k$ in the event tie ${ }_{i j}$ can be written as $\delta_{i j}(\tau)=\tau_{i} \phi_{i}+\tau_{i j} \phi_{i j}-\tau_{j k} \phi_{j k}+\tau_{k} \phi_{k}$.
} 
If conditionally on tie $i_{j}$, the expected score of $k$ is below that of $i$, the probability of $\operatorname{piv}_{i j}$ (i.e. $s_{i}=s_{j}>s_{k}$ ) is equivalent to that of the $\operatorname{tie}_{i j}$. On the other hand, if conditionally on tie ${ }_{i j}$, the expected score of $k$ is above that of $i$, then $\operatorname{piv}_{i j}$ becomes much less likely than tie $i j$, and in fact equivalent to tie abc $_{\text {. }}$

In the sequel, we write $\mu_{a b c}=\mu\left[\right.$ tie $\left._{a b c}\right]$ and, for each pair $i j$ of candidates, $\mu_{i j}=\mu\left[\operatorname{piv}_{i j}\right]$.

\section{Stable EqUilibria}

\subsection{Stable equilibria are discriminatory}

We establish a first result on the form of stable equilibria.

Theorem 2. Let $\sigma$ be a stable equilibrium. Then, $\sigma$ is discriminatory: $\mu_{a b}>\mu_{a c}, \mu_{b c}$, up to $a$ relabelling of the candidates.

In a discriminatory equilibrium, ${ }^{7}$ voters perceive that one pivot event is infinitely more likely than any other. If $\mu_{a b}>\mu_{a c}, \mu_{b c}$, each voter approves of either $a$ or $b$, depending on whether he prefers $a$ to $b$ or the opposite (and can of course approve of $c$ as well). As preferences are strict, no voter approves both $a$ and $b$ (i.e. votes $a b$ ) or disapprove them both (i.e. votes $c$ ), so that $\tau_{a b}=\tau_{c}=0$. Building on this observation, the following proposition provides formulas for the magnitudes of each pivot event in any stable equilibrium.

Proposition 2. Take any ballot profile $\tau$ with $\tau_{a b}=\tau_{c}=0$. Letting

$$
\mu_{a b c}=-\left(\sqrt{\tau_{a}}-\sqrt{\tau_{b c}}\right)^{2}-\left(\sqrt{\tau_{b}}-\sqrt{\tau_{a c}}\right)^{2},
$$

the magnitudes of the different pivot events are given by:

$$
\begin{aligned}
& \mu_{a b}=\left\{\begin{array}{cl}
-\left(\sqrt{\tau_{a}+\tau_{a c}}-\sqrt{\tau_{b}+\tau_{b c}}\right)^{2} & \text { if } \tau_{a c} \tau_{b c}<\tau_{a} \tau_{b}, \\
\mu_{a b c} & \text { if } \tau_{a c} \tau_{b c} \geq \tau_{a} \tau_{b},
\end{array}\right. \\
& \mu_{a c}=\left\{\begin{array}{cl}
-\left(\sqrt{\tau_{a}}-\sqrt{\tau_{b c}}\right)^{2} & \text { if } \tau_{b}<\tau_{a c}, \\
\mu_{a b c} & \text { if } \tau_{b} \geq \tau_{a c},
\end{array}\right. \\
& \mu_{b c}=\left\{\begin{array}{cll}
-\left(\sqrt{\tau_{b}}-\sqrt{\tau_{a c}}\right)^{2} & \text { if } \tau_{a}<\tau_{b c}, \\
\mu_{a b c} & \text { if } \tau_{a} \geq \tau_{b c} .
\end{array}\right.
\end{aligned}
$$

Proposition 2 is a central tool for our analysis of stable equilibria. As a first observation, the conditions for all two-candidate pivot magnitudes being jointly above $\mu_{a b c}$ are together incompatible, so that we always have

$$
\mu_{a b c}=\min \left\{\mu_{a b}, \mu_{a c}, \mu_{b c}\right\} .
$$

From this, we conclude that there are two sorts of stable equilibria: direct equilibria for which $\mu_{a b}>\mu_{a c}>\mu_{b c}=\mu_{a b c}$, and indirect equilibria for which $\mu_{a b}>\mu_{a c}=\mu_{b c}=\mu_{a b c}$, up to a relabelling of the candidates. While best responses can be straightforwardly inferred from the magnitude ranking in a direct equilibrium, they may depend on precise pivot probability ratios and on cardinal utilities in an indirect equilibrium.

\footnotetext{
${ }^{7}$ Note that Myerson [2002] defines a discriminatory equilibrium as one in which a pivot (say $a b$ ) is infinitely more likely than the other ones, and this does not imply that pivot magnitudes are different a priori. However, we show in the appendix (Section C.3) that the two definitions coincide for stable equilibria.
} 


\subsection{Stable equilibria violate the ordering conditions}

Before delving into the description of stable equilibria, we comment on the relation between candidates' expected scores and the relative likelihood of pivot events, which differs markedly in this game with respect to alternative models of strategic voting.

If candidates' scores were independent, one might expect that (i) pivot events involving candidates with higher expected scores should be infinitely more likely and (ii) a tie between the three candidates should be infinitely less likely than a pivot between any two candidates. These intuitive properties, known as the ordering conditions, are the main ingredients of the early strategic voting model of Myerson and Weber [1993], and can be expressed as follows.

$$
\text { (ordering conditions) } \quad\left\{\begin{array}{l}
(i) \quad \gamma_{i}>\gamma_{j} \Rightarrow \mu_{i k}>\mu_{j k} \\
(i i) \quad \forall i, j \in \mathscr{K}, \quad \mu_{i j}>\mu_{a b c}
\end{array}\right.
$$

We know from a counter-example in Núñez [2010] that condition (i) may be violated in Poisson games under AV, as candidates' scores can be correlated when some voters approve more than one candidate. We generalize this observation and obtain the surprising result that the condition is violated at any stable equilibrium.

Theorem 3. Every stable equilibrium violates the ordering conditions. More precisely,

1. Any direct equilibrium with $\mu_{a b}>\mu_{a c}>\mu_{b c}$ (up to a relabelling of the candidates), satisfies $\gamma_{a}>\gamma_{c}>\gamma_{b}$. The ordering condition (i) is violated as $\gamma_{c}>\gamma_{b}$ and yet $\mu_{a b}>\mu_{a c}$.

2. Any indirect equilibrium with $\mu_{a b}>\mu_{a c}=\mu_{b c}$ (up to a relabelling of the candidates), satisfies $\gamma_{a}, \gamma_{b} \geq \gamma_{c}$. The ordering condition (i) is violated whenever $\gamma_{a} \neq \gamma_{b}$, as $\mu_{a c}=\mu_{b c}$.

3. Any stable equilibrium violates the ordering condition (ii) as $\mu_{a b c}=\min \left\{\mu_{a b}, \mu_{a c}, \mu_{b c}\right\}$.

The result is important as it suggests that, for the analysis of strategic voting under AV, the model of Poisson games is fundamentally different from models where the ordering conditions prevail. It appears that the ordering conditions are useful but somewhat arbitrary belief restrictions, that do not capture the real relative likelihood of pivot events obtained in models where pivot probabilities are endogenous. In the sequel, we show how to characterize equilibria in such a model, without imposing any belief restriction.

\section{Direct Equilibria}

This section considers the simplest stable equilibria: direct equilibria, in which the magnitudes of pivot events fully determine voters' best responses. We first characterize preference profiles admitting a direct equilibrium, for a given ordering of pivot magnitudes. Then, we show that many preference profiles do not admit any direct equilibrium.

\subsection{Equilibrium Characterization.}

In order to illustrate the logic of direct equilibria in any preference ordering, assume for instance that $\mu_{a b}>\mu_{a c}>\mu_{b c}$. In any such equilibrium, the strict ordering of the magnitudes implies that:

$$
\mathbb{P}\left[\operatorname{piv}_{a b} \mid n \tau\right] \gg_{n \rightarrow \infty} \mathbb{P}\left[\operatorname{piv}_{a c} \mid n \tau\right] \gg_{n \rightarrow \infty} \mathbb{P}\left[\operatorname{piv}_{b c} \mid n \tau\right], \mathbb{P}\left[\operatorname{piv}_{a b c} \mid n \tau\right]
$$


In turn, this implies that best responses are uniquely determined when $n$ is large, and that cardinal utilities do not matter. The best responses for each type are represented in Figure 3, where, for instance, the arrow from $r_{a b}$ to $a$ means that $\operatorname{BR}(a b \mid n \tau)=a$.

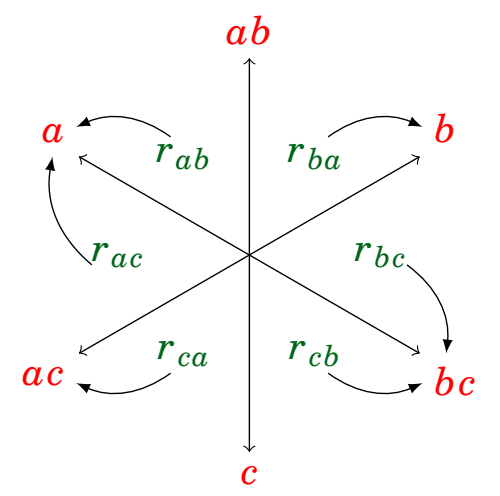

$$
\begin{aligned}
& \operatorname{BR}(a b \mid n \tau)=\operatorname{BR}(a c \mid n \tau)=a \\
& \operatorname{BR}(b c \mid n \tau)=\operatorname{BR}(c b \mid n \tau)=b c \\
& \operatorname{BR}(b a \mid n \tau)=b \\
& \operatorname{BR}(c a \mid n \tau)=a c
\end{aligned}
$$

FIGURE 3. Best responses (for $n$ large) in a direct equilibrium with $\mu_{a b}>\mu_{a c}>\mu_{b c}$.

In words, as pivot $a b$ dominates, each voter approves of his favorite candidate between $a$ and $b$. Next, as pivot $a c$ comes second, a voter approves of $c$ if and only if he prefers $c$ to $a$. It follows that

$$
\tau_{a}=r_{a b}+r_{a c}, \quad \tau_{b}=r_{b a}, \quad \tau_{a c}=r_{c a}, \quad \tau_{b c}=r_{b c}+r_{c b}, \quad \tau_{c}=\tau_{a b}=0
$$

The constructed strategy profile $\sigma$ is indeed a direct equilibrium if the associated ballot profile $\tau$ triggers the magnitude ordering $\mu_{a b}>\mu_{a c}>\mu_{b c}$.

As $\tau_{a b}=\tau_{c}=0$, Proposition 2 implies that the magnitude ordering is indeed satisfied if and only if:

$$
\frac{\tau_{a}}{\tau_{b c}}>\frac{\tau_{a c}}{\tau_{b}}>1 \quad \text { and } \quad-\left(\sqrt{\tau_{a}+\tau_{a c}}-\sqrt{\tau_{b}+\tau_{b c}}\right)^{2}>-\left(\sqrt{\tau_{a}}-\sqrt{\tau_{b c}}\right)^{2} . \quad(* *)
$$

To conclude, we obtained a characterization of direct equilibria: ${ }^{8}$ a preference profile $r$ admits a direct equilibrium if and only if conditions $(*)$ and $(* *)$ can be jointly satisfied for some labelling of the candidates.

\subsection{Existence}

In this section we show that, for many preference profiles, a direct equilibrium does not exist. To see why, observe that, by combining conditions $(*)$ and $(* *)$, we obtain that a necessary condition to have a direct equilibrium with $\mu_{a b}>\mu_{a c}>\mu_{b c}$ is to have:

$$
\frac{r_{a}}{r_{-a}}>\frac{r_{c a}}{r_{b a}}>1
$$

where $r_{a}$ denotes the share of voters who rank $a$ first and $r_{-a}$ denotes the share of voters who rank $a$ last.

Consider the preference profile $r$, denoting a symmetric Condorcet cycle: $r_{a b}=r_{b c}=r_{c a}=1 / 3$ so that $r_{k} / r_{-k}=1$ for $k=a, b, c$. It is then immediate to see that the preference profile $r$ does not admit a direct equilibrium. By extension, we can prove that the non-existence of a direct

\footnotetext{
${ }^{8}$ Note that direct equilibria are indeed stable, since the magnitude ordering remains the same in a neighborhood of $\tau$, by virtue of condition $(* *)$.
} 
equilibrium remains true in a neighborhood of this preference profile. We thus obtain the following result.

Proposition 3. The set of preference profiles admitting no direct equilibrium is of positive measure.

This result is important for the analysis of Poisson voting games: in many cases, there is no equilibrium with a strict pivot magnitude ordering. This implies that we often need to look beyond pivot magnitudes to derive voters' best responses, a step that is undertaken in Section 5.

\subsection{Quantitative Assessment}

To assess quantitatively the prevalence of direct equilibria, we performed Monte-Carlo simulations consisting of 100,000 draws of the preference profile $r$, the draws being independent and uniform on the simplex over $\mathscr{T}$.

As a first observation, we note that direct equilibria exist in $27.7 \%$ of the preference profiles. This reinforces the conclusion of Proposition 3: only looking at direct equilibria would not allow to understand strategic behavior under $\mathrm{AV}$ in a large number of situations.

To go further, we would like to identify preference profiles for which direct equilibria exist (or do not). As a first step, we might hint from earlier models operating with the ordering conditions that direct equilibria are unlikely to be found when a Condorcet cycle exists. ${ }^{9}$ Numerically, we find that this intuition is almost exact: there appears to be around $0.1 \%$ of preference profiles admitting a Condorcet cycle for which a direct equilibrium exists.

When the preference profile admits a Condorcet winner, we observe that a direct equilibrium exists only for $29.8 \%$ of the preference profiles (as a reference, such an equilibrium always exists under the ordering conditions). Focusing on the candidate that can be elected at a direct equilibrium, we observe that it is almost always the Condorcet winner. More precisely, 29.7\% of the profiles admit a direct equilibrium electing the Condorcet winner, but no direct equilibrium electing another candidate. Less than $0.1 \%$ of the profiles admit a direct equilibrium electing a non-Condorcet winner, but no direct equilibrium electing the Condorcet winner. ${ }^{10}$ Finally, only 3 profiles over our 100,000 draws admit both a direct equilibrium electing the Condorcet winner, and another direct equilibrium electing another candidate.

\subsection{An Example of Direct Equilibrium}

We consider an electorate composed of three types of voters: $a b, b a$ and $c a$ with respective shares

$$
r_{a b}=\frac{3}{10}, r_{b a}=\frac{3}{10} \text { and } r_{c a}=\frac{4}{10} \text {. }
$$

9The logic is quite straightforward under the ordering conditions. As argued in the introduction, any equilibrium with $\gamma_{a}>\gamma_{b}>\gamma_{c}$ satisfying the ordering conditions is such that

$$
\gamma_{a}=\sum_{t: a>b} r_{t}, \gamma_{b}=\sum_{t: b>a} r_{t} \quad \text { and } \gamma_{c}=\sum_{t: c>a} r_{t} .
$$

As $\gamma_{a}>\gamma_{b}$, we obtain that a majority prefers $a$ to $b$, so that $\gamma_{a}>1 / 2>\gamma_{b}$. As we have a Condorcet cycle, a majority prefers $c$ to $a$, so that $\gamma_{c}>1 / 2$, hence a contradiction with $\gamma_{b}>\gamma_{c}$.

10 Such a situation, with both one equilibrium electing the Condorcet Winner and one not electing him, was first presented in Núñez [2010]. 
Since we look for a direct equilibrium, preference intensities do not play any role and we leave the values $u_{a b}, u_{b a}$ and $u_{c a}$ unspecified. This preference profile admits a unique direct equilibrium $\sigma$ with

$$
\sigma_{a b}=a, \sigma_{b a}=b \text { and } \sigma_{c a}=a c .
$$

The strategy profile $\sigma$ is depicted in Figure 4, together with candidates' expected scores.

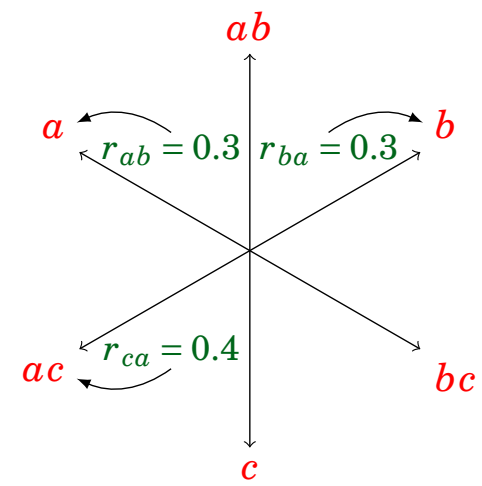

(A) Strategies of the voters.

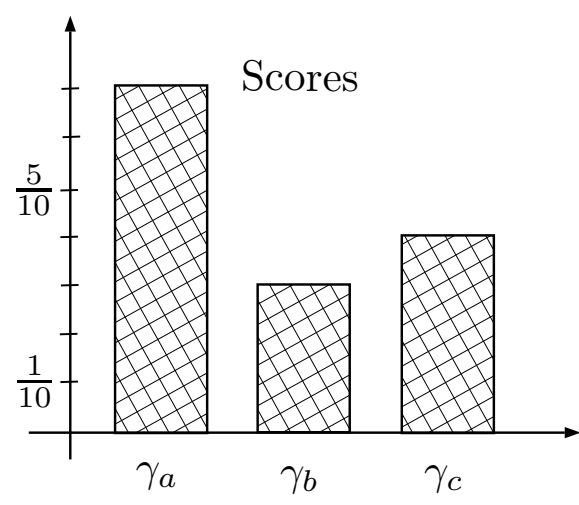

(B) Candidates expected scores.

FIGURE 4. A direct equilibrium with $\mu_{a b}>\mu_{a c}>\mu_{b c}$.

The strategy profile thus leads to the victory of the Condorcet winner $a$. As $\tau_{c}=\tau_{a b}=0$, we may apply the formulas in Proposition 2, and we obtain $\mu_{a b}>\mu_{a c}>\mu_{b c}$ since

$$
\begin{array}{lll}
\mu_{a b}=-0.0835 & \text { with } & \tau_{a c} \tau_{b c}=0<\tau_{a} \tau_{b}, \\
\mu_{a c}=-0.3 & \text { with } \quad \tau_{b}<\tau_{a c}, \\
\mu_{b c}=\mu_{a b c}=-0.3071 & \text { with } & \tau_{b c}=0<\tau_{a} .
\end{array}
$$

As we observed earlier, the ordering condition (i) is violated in this example, as $\mu_{a b}$ is maximal, but candidates $a$ and $c$ have the two highest scores. Note that this example does not hinge of the precise type distribution, as, for each $0 \leq \eta<3$, letting $r^{\eta}$ denote the type distribution with

$$
r_{a b}^{\eta}=\frac{3+\eta}{10}, r_{b a}^{\eta}=\frac{3-\eta}{10} \text { and } r_{c a}^{\eta}=\frac{4}{10},
$$

the strategy profile $\sigma$ is a direct equilibrium since, for each $0 \leq \eta<3$,

$$
\mu_{a b}=-\left(\sqrt{\frac{7+\eta}{10}}-\sqrt{\frac{3-\eta}{10}}\right)^{2}>\mu_{a c}=-\frac{3+\eta}{10}>\mu_{b c}=\mu_{a b c}=-\frac{3+\eta}{10}-\left(\sqrt{\frac{3-\eta}{10}}-\sqrt{\frac{4}{10}}\right)^{2}
$$

\section{INDIRECT EQUILIBRIA}

This section considers the second kind of stable equilibria: indirect equilibria in which the magnitudes of pivot events do not fully determine voters' best responses. We first show how to derive best responses by estimating the likelihood ratios of pivot events. Then, we provide a general existence result: for any ordinal preference profile, there exists a specification of cardinal preferences for which an indirect equilibrium exists. 


\subsection{Equilibrium Characterization}

Let $\sigma$ be a strategy profile such that $\mu_{a b}>\mu_{a c}=\mu_{b c}=\mu_{a b c}$. Looking at Proposition 2, a ballot profile $\tau$ generates this ordering of magnitudes if and only if $\tau_{b} \geq \tau_{a c}, \tau_{a} \geq \tau_{b c}$ and $\tau_{a} \tau_{b}>\tau_{a c} \tau_{b c}$. As illustrated on Figure 5, the best response is fully determined for types $a b, b a, c b$ and $c a$ (solid arrows), because the pivot $a b$ dominates. However, types $a c$ and $b c$ need to assess the relative likelihood of pivot events $a c, b c$ and $a b c$ : their best response is indeterminate (dashed arrows).

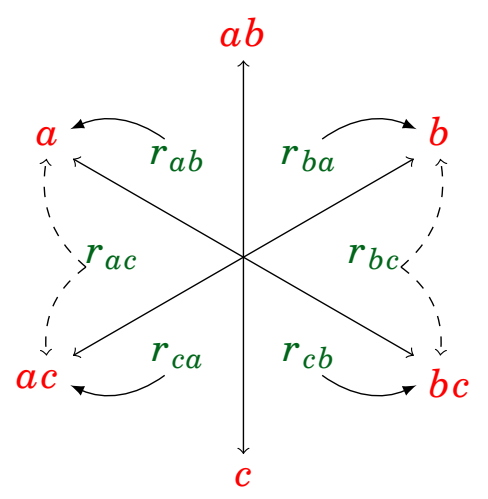

FIGURE 5. Best responses (for $n$ large) in an indirect equilibrium with $\mu_{a b}>\mu_{a c}=\mu_{b c}$.

To determine the best responses of types $a c$ and $b c$, we compute for each type the relative probabilities of pivots $a c, b c$ and tie $a b c$. A central tool for this analysis is the notion of an offset ratio. Intuitively, the offset ratio $\phi_{m}$ of a ballot $m$ at an event $E$ corresponds to the most likely share of ballots $m$ when $E$ occurs, divided by its expected value $n \tau_{m}$. As shown in the next result, the offset ratios in the event tie ${ }_{a b c}$ constitute sufficient statistics to compute the relative likelihood of any relevant pivot event with respect to tie ${ }_{a b c}$.

The values of these offset ratios are obtained as follows. Applying the Dual Magnitude Theorem [Myerson, 2002], the magnitude $\mu\left[\operatorname{tie}_{a b c}\right.$ ] equals the optimal value of

$$
\min _{x, y \in \mathbb{R}} \tau_{a} e^{x+y}+\tau_{a c} e^{x}+\tau_{b} e^{-x}+\tau_{b c} e^{-(x+y)}-1 .
$$

Moreover, denoting by $\left(x^{*}, y^{*}\right)$ the values of $x, y$ at the optimum, the offset ratios are given by:

$$
\phi_{a}=e^{x^{*}+y^{*}}=\sqrt{\frac{\tau_{b c}}{\tau_{a}}}, \quad \phi_{b}=e^{-x^{*}}=\sqrt{\frac{\tau_{a c}}{\tau_{b}}}, \quad \phi_{a c}=1 / \phi_{b} \quad \text { and } \quad \phi_{b c}=1 / \phi_{a} .
$$

The following result derives the relative likelihood of pivot and tie events and characterizes indirect equilibria.

Theorem 4. Let $\sigma$ be a stable equilibrium such that $\mu_{a b}>\mu_{a c}=\mu_{b c}$ (indirect equilibrium). Then, for $t \in\{a c, b c\}$ :

1. The probabilities of pivot and tie events satisfy:

$$
\begin{aligned}
& \forall\{i, j\}=\{a, b\}, \quad \lim _{n \rightarrow \infty} \frac{\mathbb{P}\left[\operatorname{piv}_{i c}^{t} \mid n \tau\right]}{\mathbb{P}\left[\operatorname{tie}_{a b c} \mid n \tau\right]}=\frac{\phi_{j}}{1-\phi_{j}}\left(\phi_{t_{1}}+\phi_{t_{1} t_{2}}\right), \\
& \lim _{n \rightarrow \infty} \frac{\mathbb{P}\left[\mathrm{tie}_{a b c}^{t, 1} \mid n \tau\right]}{\mathbb{P}\left[\mathrm{tie}_{a b c} \mid n \tau\right]}=\phi_{t_{1}}, \\
& \lim _{n \rightarrow \infty} \frac{\mathbb{P}\left[\mathrm{tie}_{a b c}^{t, 2} \mid n \tau\right]}{\mathbb{P}\left[\mathrm{tie}_{a b c} \mid n \tau\right]}=\phi_{t_{1} t_{2}} .
\end{aligned}
$$


2. There exists a cutoff $u_{t}^{*} \in(0,1)$ such that

$$
\left\{\begin{array}{l}
u_{t}<u_{t}^{*} \Rightarrow \sigma_{t}=t_{1} \\
u_{t}>u_{t}^{*} \Rightarrow \sigma_{t}=t_{1} t_{2}
\end{array}\right.
$$

and given by

$$
u_{t}^{*}=\frac{f_{t}\left(\phi_{t_{1} t_{2}}\right)}{f_{t}\left(\phi_{t_{1} t_{2}}\right)+f_{t}\left(1 / \phi_{t_{1}}\right)} \quad \text { with } \quad f_{t}(y)=\phi_{t_{1}}+\left(\phi_{t_{1}}+\phi_{t_{1} t_{2}}\right)\left(\frac{y+2}{y-1}\right)
$$

where the offset ratios are the ones associated to tie $\mathrm{abc}_{\text {c }}$.

\subsection{Existence and Condorcet consistency}

Contrary to direct equilibria, we establish that indirect equilibria exist for any ordinal preference profile $r$ (at least for some values of the preference intensities). Moreover, we obtain that the equilibrium we construct elects the Condorcet winner when he exists, a property referred to as Condorcet consistency.

Let $r$ be an ordinal preference profile and let $a$ be a candidate such that $r_{a}>r_{-a}$ (the share of voters who rank $a$ first is larger than the share of voters who rank $a$ last). Let $b$ be a candidate such that $r_{b a}>r_{c a} \cdot{ }^{11}$ We will show that there exists some $u \in(0,1)^{\mathscr{T}}$ for which an equilibrium exists with $\mu_{a b}>\mu_{a c}=\mu_{b c}$. Let $\sigma$ be the strategy profile defined by:

$$
\sigma_{a b}=\sigma_{a c}=a, \quad \sigma_{b c}=\sigma_{c b}=b c, \quad \sigma_{b a}=b \quad \text { and } \quad \sigma_{c a}=a c .
$$

Under this strategy profile, we know that $\tau_{a}=r_{a}, \tau_{b}=r_{b a}, \tau_{a c}=r_{c a}, \tau_{b c}=r_{-a}$ and $\tau_{c}=\tau_{a b}=$ 0 . By assumption, we thus have $\tau_{a}>\tau_{b c}, \tau_{b}>\tau_{a c}$ and $\tau_{a} \tau_{b}>\tau_{a c} \tau_{b c}$. Therefore, we obtain from Proposition 2 that the ordering $\mu_{a b}>\mu_{a c}=\mu_{b c}$ is indeed satisfied under the strategy profile $\sigma$.

From the magnitude ordering, we know directly that $\sigma_{t}=\mathrm{BR}(t \mid n \tau)$ for any type $t \in\{a b, b a, c a, c b\}$. Following the proof of Theorem 4, there exists for each $t \in\{a c, b c\}$ a number $u_{t}^{*} \in(0,1)$ such that, for $n$ large enough: $u_{t}<u_{t}^{*} \Rightarrow \mathrm{BR}(t \mid n \tau)=t_{1}$ and $u_{t}>u_{t}^{*} \Rightarrow \mathrm{BR}(t \mid n \tau)=t_{1} t_{2}$. We conclude that, for $u_{a c}$ small enough and $u_{b c}$ large enough, the strategy profile $\sigma$ is an indirect equilibrium. ${ }^{12}$

Finally, let us consider the case where a profile $r$ admits a Condorcet winner, say $a$, and let us show that $r_{a}>r_{-a}$. As a majority prefers $a$ to $b$, we have that $r_{a}+r_{c a}>r_{-a}+r_{b a}$; as a majority prefers $a$ to $c$, we have that $r_{a}+r_{b a}>r_{-a}+r_{c a}$. Thus, $r_{a}-r_{-a}>\left|r_{c a}-r_{b a}\right|>0$. Since $\gamma_{a}=r_{a}+r_{c a}, \gamma_{b}=r_{-a}+r_{b a}$ and $\gamma_{c}=r_{-a}+r_{c a}$, the candidate $a$ is elected, and we thus obtain the following result.

Proposition 4. For any ordinal preference profile $r$, there exists an open set of cardinal representations $u \in(0,1)^{\mathscr{T}}$ such that an indirect equilibrium exists. If $r$ admits a Condorcet winner, it is elected at the constructed equilibrium.

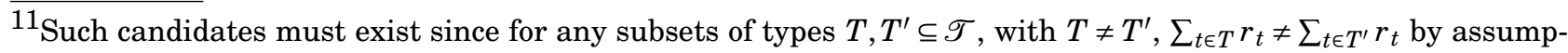
tion.

${ }^{12}$ Note that the magnitude ordering is preserved in a neighborhood of $\tau$, and that each $u_{t}^{*}$ is a continuous function of $\tau$. Thus, the strategy profile $\sigma$ is indeed a stable equilibrium.
} 


\subsection{Quantitative Assessment}

As for direct equilibria, we conducted Monte-Carlo simulations to assess indirect equilibria. Building on the equilibrium characterization in Theorem 4, we can describe the set of cardinal preferences allowing the existence of any potential indirect equilibrium, for each ordinal preference profile $r$. Thus, we first draw 200,000 ordinal preference profiles $r$ : 100,00 draws uniformly on the subset of the simplex on $\mathscr{T}$ for which a Condorcet winner exists; and 100,000 draws uniformly on the subset of the simplex on $\mathscr{T}$ for which a Condorcet cycle exists. Then, for each draw, we can compute explicitly the probability of existence (resp. of co-existence) of any indirect equilibrium (resp. of any set of indirect equilibria), assuming that utilities $\left(u_{t}\right)_{t \in \mathscr{T}}$ are drawn uniformly on [0,1], and independently across types.

We first present the distribution of the probabilities of existence of a stable equilibrium over the universe of ordinal preference profiles, when a Condorcet winner exists. This distribution is plotted in Figure 6. We observe on the right the $29.8 \%$ of ordinal preference profiles for which an equilibrium exists with probability one, which corresponds to direct equilibria (note that indirect equilibria may also exist at these profiles). For all other ordinal preference profiles, we observe that an (indirect) equilibrium exists with strictly positive probability, as shown in Proposition 4. Furthermore, we see that this probability of existence is significant, above $30 \%$ for most ordinal preference profiles.

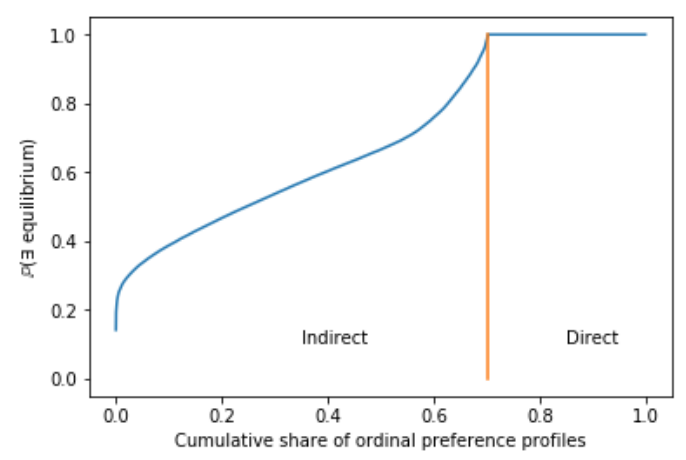

FIGURE 6. Distribution of the probabilities of existence of a stable equilibrium when a Condorcet winner exists

As we know that direct and indirect equilibria may coexist at a given preference profile, one may wonder whether different candidates may be elected for the same profile. We draw on Figure 7 the distribution of the number of candidates that can be elected at a stable equilibrium for a given (cardinal) preference profile, when a Condorcet winner exists. We observe that whenever a stable equilibrium exists (at least one possible winner), it is very rare that 2 candidates be potential winners of the election (1.9\% of all cardinal preference profiles).

Then, we investigate whether the likely winner of the election is the Condorcet winner or not. To that aim, we plot on Figure 8 two distributions: the probabilities of existence of a stable equilibrium electing the Condorcet winner (resp. electing a non-Condorcet winner) over the universe of ordinal preference profiles, when a Condorcet winner exists. We observe that for most ordinal preference profiles, only a Condorcet winner can be elected. For around 15\% of all ordinal profiles, a non-Condorcet winner may be elected, but with a small probability, 


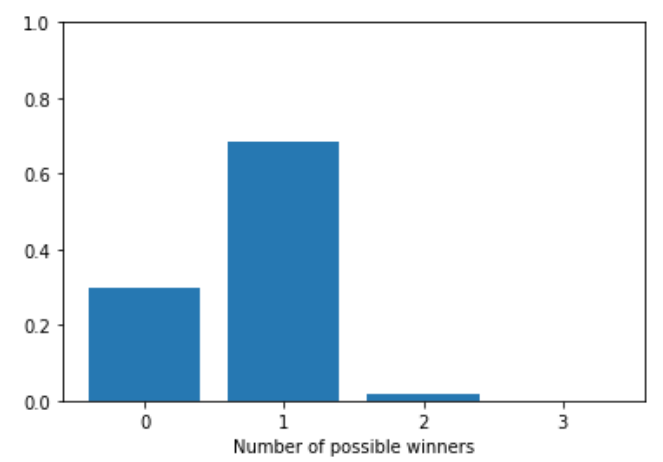

FIGURE 7. Distribution of the number of possible winners at a stable equilibrium when a Condorcet winner exists

typically smaller than the probability with which there exists a stable equilibrium electing the Condorcet winner.

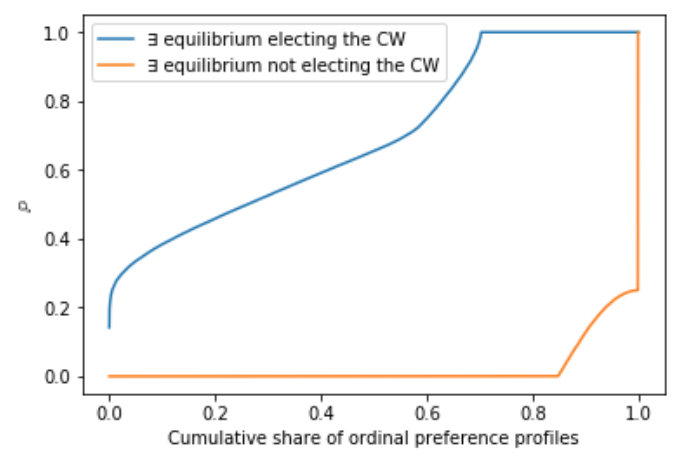

FIGURE 8. Distributions of the probabilities of existence of a stable equilibrium electing the Condorcet winner (resp. electing a non-Condorcet winner), when a Condorcet winner exists

Finally, we consider profiles for which no Condorcet winner exists: there is a Condorcet cycle. We report on Figure 9 the distribution of the probabilities of existence of an equilibrium for these preference profiles. We observe that there always is a significant probability that an (indirect) equilibrium exists. This can be seen as an improvement over earlier models which didn't provide any prediction for these preference profiles [Laslier, 2009].

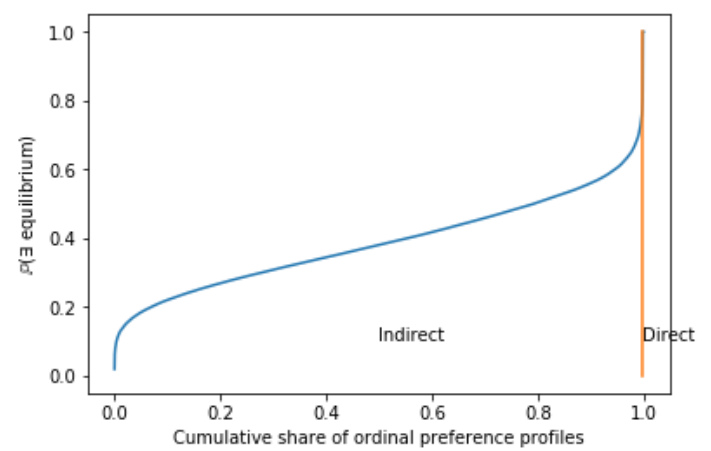

FIGURE 9. Distribution of the probabilities of existence of an equilibrium when a Condorcet cycle exists 
Finally, we report on Figure 10 the distribution of the number of candidates that can be elected at a stable equilibrium for a given (cardinal) preference profile, when a Condorcet cycle exists. We observe that when a stable equilibrium exists, the possible winner is most likely unique. Moreover, there are rare cases for which any of the three candidates may be elected at a stable equilibrium (for around $1.9 \%$ of all cardinal preference profiles).

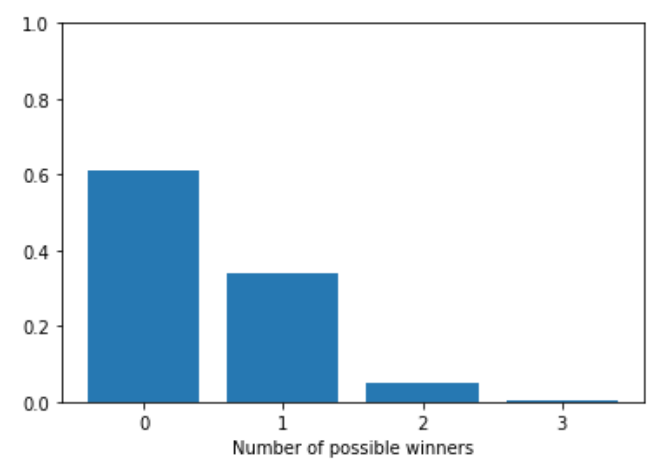

FIGURE 10. Distribution of the number of possible winners at a stable equilibrium when a Condorcet cycle exists

\subsection{An Example of Indirect Equilibrium}

We consider an electorate composed of four types of voters to illustrate indirect equilibria characterized in the previous section: $a b, b c, c a$ and $c b$ with respective shares

$$
r_{a b}=\frac{3}{10}, \quad r_{b c}=\frac{3}{10}, \quad r_{c a}=\frac{2+\eta}{10} \quad \text { and } r_{c b}=\frac{2-\eta}{10},
$$

with $0 \leq \eta<1$. The equilibrium that we analyse does not depend on $u_{a b}, u_{c a}$ and $u_{c b}$. Yet, $u_{b c}$ remains to be specified since best responses depend on its precise value. It can be shown that this preference profile does not admit any direct equilibrium since conditions $(*)$ and $(* *)$ cannot be jointly satisfied for any labelling of the candidates.

This preference profile admits an indirect equilibrium $\sigma$ defined by

$$
\sigma_{a b}=a, \quad \sigma_{b c}=b, \quad \sigma_{c a}=a c \quad \text { and } \sigma_{c b}=b c .
$$

The strategy profile $\sigma$ is depicted in Figure 11, together with candidates' corresponding expected scores.

The strategy profile $\sigma$ leads to the victory of $a$ since $\gamma_{a}>\gamma_{b}>\gamma_{c}$ for any $\eta$. As $\tau_{a b}=\tau_{c}=0$, pivot magnitudes can be computed as in Proposition 2. We obtain:

$$
\begin{aligned}
& \mu_{a b}=\frac{\sqrt{(5+\eta)(5-\eta)}}{5}-1 \quad \text { since } \quad \tau_{a c} \tau_{b c}<\tau_{a} \tau_{b}, \\
& \mu_{a c}=\mu_{b c}=\mu_{a b c}=\frac{\sqrt{3(2+\eta)}+\sqrt{3(2-\eta)}}{5}-1 \quad \text { since } \tau_{b} \geq \tau_{a c} \text { and } \tau_{a} \geq \tau_{b c} .
\end{aligned}
$$

One can check that $\mu_{a b}>\mu_{a c}=\mu_{b c}=\mu_{a b c}$ for any $0 \leq \eta<1$ as required. Building on Proposition 1 , the best responses of the different types of voters are as follows: ( $i$ ) $a b$-voters approve only $a$ since $\mu_{a b}$ is maximal , ( $\left.i i\right) c a$-voters approve of $c$ since $c$ is their most preferred candidate and approve of $a$ since $\mu_{a b}$ is maximal and (iii) $c b$-voters approve of $c$ since $c$ is their most preferred candidate and approve of $b$ since $\mu_{a b}$ is maximal. 


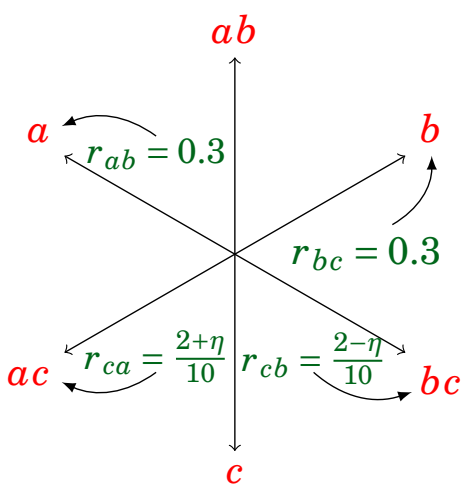

(A) Strategies of the voters.

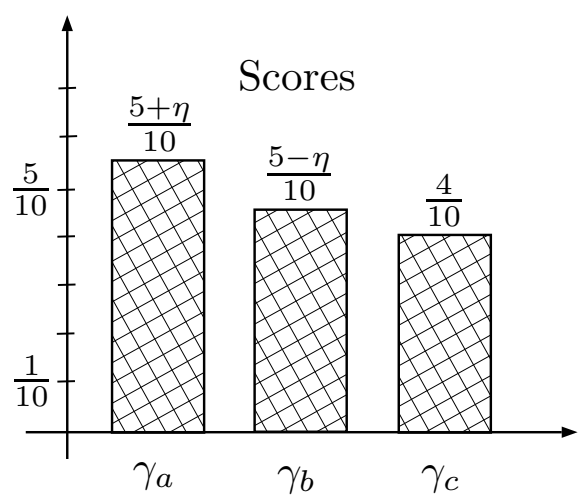

(B) Candidates' expected scores.

FIGURE 11. An indirect equilibrium with $\mu_{a b}>\mu_{a c}=\mu_{b c}$

The decision of $b c$-voters relies on the exact relative likelihoods of pivot events, which are obtained from the offset ratios. Recall that, for $t=b c, \Delta(t \mid n \tau)$ equals:

$$
\mathbb{P}\left[\operatorname{piv}_{a c}^{t} \mid n \tau\right]\left(\frac{1-u_{b c}}{2}\right)+\mathbb{P}\left[\operatorname{piv}_{b c}^{t} \mid n \tau\right]\left(\frac{-u_{b c}}{2}\right)+\left(2 \mathbb{P}\left[\operatorname{tie}_{a b c}^{t, 1} \mid n \tau\right]+\mathbb{P}\left[\operatorname{tie}_{a b c}^{t, 2} \mid n \tau\right]\right)\left(\frac{1-2 u_{b c}}{6}\right) .
$$

As shown by Theorem 4, the limit ratio of pivot probabilities equals, for $t=b c$,

$$
\begin{array}{ll}
\lim _{n \rightarrow \infty} \frac{\mathbb{P}\left[\operatorname{piv}_{a c}^{t} \mid n \tau\right]}{\mathbb{P}\left[\operatorname{tie}_{a b c} \mid n \tau\right]}=\frac{\phi_{b}}{1-\phi_{b}}\left(\phi_{b}+\phi_{b c}\right), & \lim _{n \rightarrow \infty} \frac{\mathbb{P}\left[\operatorname{piv}_{b c}^{t} \mid n \tau\right]}{\mathbb{P}\left[\operatorname{tie}_{a b c} \mid n \tau\right]}=\frac{\phi_{a}}{1-\phi_{a}}\left(\phi_{b}+\phi_{b c}\right), \\
\lim _{n \rightarrow \infty} \frac{\mathbb{P}\left[\operatorname{tie}_{a b c}^{t, 1} \mid n \tau\right]}{\mathbb{P}\left[\operatorname{tie}_{a b c} \mid n \tau\right]}=\phi_{b}, & \lim _{n \rightarrow \infty} \frac{\mathbb{P}\left[\operatorname{tie}_{a b c}^{t, 2} \mid n \tau\right]}{\mathbb{P}\left[\operatorname{tie}_{a b c} \mid n \tau\right]}=\phi_{b c} .
\end{array}
$$

where the offset ratios are given by:

$$
\phi_{a}=\sqrt{\frac{\tau_{b c}}{\tau_{a}}}=\sqrt{\frac{2-\eta}{3}}, \quad \phi_{b}=\sqrt{\frac{\tau_{a c}}{\tau_{b}}}=\sqrt{\frac{2+\eta}{3}}, \phi_{a c}=\sqrt{\frac{3}{2+\eta}} \quad \text { and } \quad \phi_{b c}=\sqrt{\frac{3}{2-\eta}} .
$$

Therefore, we can conclude that $\operatorname{BR}(b c \mid n \tau)=b$ if and only if $u_{b c}<u^{*}(\eta)$ with

$$
u^{*}(\eta)=\frac{\phi_{b}+\left(\phi_{b}+\phi_{b c}\right)\left(\frac{\phi_{b c}+2}{\phi_{b c}-1}\right)}{2 \phi_{b}+\left(\phi_{b}+\phi_{b c}\right)\left(\frac{\phi_{b c}+2}{\phi_{b c}-1}+\frac{\phi_{a c}+2}{\phi_{a c}-1}\right)} .
$$

It can be shown that $u^{*}(0)=0.5, u^{*}(1)=0$ and that $u^{*}(\eta)$ is decreasing in $\eta$ for $0 \leq \eta<1$. To conclude, $\sigma$ is an equilibrium as long as $u_{b c}<u^{*}(\eta)$.

As shown in Theorem 3, the indirect equilibrium $\sigma$ violates the ordering condition (i), since we have $\mu_{a c}=\mu_{b c}$, and yet $\gamma_{a}>\gamma_{c}$. We even observe more precisely that the ratio $\mathbb{P}\left[\operatorname{piv}_{a c}^{t}\right.$ । $n \tau] / \mathbb{P}\left[\right.$ piv $\left._{b c}^{t} \mid n \tau\right]$ tends to a positive constant as $n$ gets large. This observation extends to indirect equilibria in general, by direct application of Theorem 4 . 


\section{CONCLUDING REMARKS}

This paper gives an in-depth account of Approval Voting with private values in the main setting for understanding strategic voting: Poisson games. It first proves that stable equilibria are discriminatory, which means that a pivot is infinitely more likely than the other ones, so that a pair of candidates focuses the attention of the electorate. It then divides stable equilibria into two main classes: direct and indirect ones. In a direct equilibrium, pivot magnitudes differ and best responses are immediate, whereas in an indirect one, some pivot magnitudes coincide which renders best responses dependent on preference intensities. Above all, the main result of this work is that no stable equilibrium satisfies the ordering conditions, a set of belief restrictions used in early works to derive predictions on AV. This violation is partly a consequence of the correlation of candidates' scores, arising under AV in Poisson games, but which does not occur in the models of Myerson and Weber [1993] and Laslier [2009]. Building on the previous description of equilibria, we have run Monte-Carlo simulations that give us global figures on the structure of stable equilibria under AV. Among the different findings, we can underline two of them.

The first finding concerns the existence of indirect equilibria. These equilibria are, to our knowledge, novel in the literature. Quantitatively, indirect equilibria are important. First, there always exists an indirect equilibrium, at least for some preference intensities, while there are many ordinal preference profiles for which no direct equilibrium exists, even for profiles admitting a Condorcet winner. Second, we observe that the actual probabilities with which an indirect equilibrium exists are significant, both when a Condorcet winner exists and when there is a Condorcet cycle.

The second finding concerns the Condorcet consistency of AV. When a Condorcet winner exists, there always exists an indirect equilibrium that elects him, at least for some preference intensities. If in addition, there exists a direct equilibrium, it is very likely that this equilibrium elects the Condorcet winner. Overall, the probabilities of having a stable equilibrium electing the Condorcet winner are much higher than having a stable equilibrium electing another candidate (Figure 8).

As a final remark on this work, we investigate what would happen in Poisson games if the correlation in candidates' scores was assumed away. We observe that our methods can be profitably exploited to analyse that case. More precisely, we introduce a behavioral twist in the analysis: voters neglect correlation, that is, they compute pivot probabilities and magnitudes as if the scores of the different candidates were independent. ${ }^{13}$ As we show in the appendix (Section B), this alternative model yields the following result.

Theorem 5. In the presence of correlation neglect, any equilibrium with $\gamma_{a}>\gamma_{b}>\gamma_{c}$ is either:

1. a direct equilibrium with $\mu_{a b}>\mu_{a c}>\mu_{b c}$, if $\gamma_{b}^{2}<\gamma_{a} \gamma_{c}$.

2. an indirect equilibrium with $\mu_{a b}>\mu_{a c}=\mu_{b c}$, if $\gamma_{b}^{2} \leq \gamma_{a} \gamma_{c}$.

\footnotetext{
${ }^{13}$ While this extension is here developed as a sanity check, the literature on correlation neglect is now firmly established in the economics literature (see Levy and Razin [2015] among others). This behavioral twist, called correlation neglect is grounded on the inability of voters to understand the consequences of correlation and is considered in the literature as a plausible restriction on beliefs. Moreover, on practical terms, it simplifies the analysis as the pivot magnitudes anticipated by voters who neglect correlation just depend on the scores of the candidates and not anymore the share of the different ballots as in the classical analysis.
} 
This result underlines that indirect equilibria exist independently of the correlation between candidates' scores.

\section{REFERENCES}

D.S Ahn and S. Oliveros. Approval voting and scoring rules with common values. Journal of Economic Theory, 166:304-310, 2016.

L. Bouton and M. Castanheira. One Person, Many Votes: Divided Majority and Information Aggregation. Econometrica, 80:43-87, 2012.

L. Bouton and G. Gratton. Majority runoff elections: strategic voting and duverger's hypothesis. Theoretical Economics, 10(2):283-314, 2015.

S. Brams. Approval voting in multicandidate elections. Policy Studies Journal, 9:102-108, 1980.

F. De Sinopoli, B. Dutta, and J-F. Laslier. Approval Voting: Three Examples. International Journal of Game Theory, 38:27-38, 2006.

M. Fey. Stability and coordination in duverger's law: A formal model of preelection polls and strategic voting. American Political Science Review, 91(1):135-147, 1997.

J. Goertz and F. Maniquet. On the Informational Efficiency of Approval Voting. Journal of Economic Theory, 146:1464-1480, 2011.

J.F. Laslier. The Leader Rule: Strategic Approval Voting in a Large Electorate. Journal of Theoretical Politics, 21:113-136, 2009.

J.F. Laslier and R. Sanver. The Basic Approval Voting Game. In J.F. Laslier and R. Sanver, editors, Handbook on Approval Voting. Heildelberg: Springer-Verlag, 2010.

G. Levy and R. Razin. Correlation Neglect, Voting Behavior, and Information Aggregation. American Economic Review, 105(4):1634-45, 2015.

R. Myerson. Population Uncertainty and Poisson Games. International Journal of Game Theory, 27:375-392, 1998.

R. Myerson. Large poisson games. Journal of Economic Theory, 94:7-45, 2000.

R. Myerson. Comparison of Scoring Rules in Poisson Voting Games. Journal of Economic Theory, 103:219-251, 2002.

R. Myerson and R.J. Weber. A theory of voting equilibria. American Political Science Review, 87:102-114, 1993.

M. Núñez. Condorcet Consistency of Approval Voting: A Counter Example on Large Poisson Games. Journal of Theoretical Politics, 22:64-84, 2010.

A. Okada. On Stability of Perfect Equilibrium Points. International Journal of Game Theory, 10(2):67-73, 1981.

T.R. Palfrey and H. Rosenthal. A Strategic Calculus of Voting. Public choice, 41(1):7-53, 1983.

T.R. Palfrey and H. Rosenthal. Voter Participation and Strategic Uncertainty. American Political Science Review, 79(1):62-78, 1985. 


\section{Appendix A. Proofs}

\section{A.1. Proof of Proposition 1}

Proof. We describe in-depth the decision problem of a voter with type $i j$ (with $k$ denoting the remaining candidate). As the type $t$ is fixed in this proof, we will write $u$ for $u_{t}$. By definition, we have

$$
U_{t}[i \mid n \tau]-U_{t}[i j \mid n \tau]=\sum_{z \in \mathcal{Z}} \mathbb{P}[z \mid n \tau]\left(U_{t}[i \mid z]-U_{t}[i j \mid z]\right) .
$$

Like we did for the particular case of two candidates, we define for each $K \subseteq \mathscr{K}$ the event $\operatorname{piv}_{K}=\left\{z \in \mathcal{Z} \mid s_{i}(z)=s_{j}(z)>s_{k}(z), \forall i, j \in K, \forall k \notin K\right\}$, where all candidates in $K$ are tied and have larger scores than the other candidates. Note that $\operatorname{piv}_{a b c}=$ tie $_{a b c}$. The expected utility of a voter with type $t$ approving of $i$ equals:

$$
\begin{gathered}
U_{t}[i \mid n \tau]=\overbrace{\mathbb{P}\left[\operatorname{piv}_{i}-i\right] \cdot 1+\mathbb{P}\left[\operatorname{piv}_{j}-i\right] \cdot u+\mathbb{P}\left[\operatorname{piv}_{k}-i\right] \cdot 0}^{\text {Single-winner events }} \\
+\overbrace{\mathbb{P}\left[\operatorname{piv}_{i j}-i\right]\left(\frac{1+u}{2}\right)+\mathbb{P}\left[\operatorname{piv}_{i k}-i\right]\left(\frac{1}{2}\right)+\mathbb{P}\left[\operatorname{piv}_{j k}-i\right]\left(\frac{u}{2}\right)}^{\text {Two-way pivots }} \\
+\overbrace{\mathbb{P}\left[\operatorname{piv}_{a b c}-i\right]\left(\frac{1+u}{3}\right)}^{\text {Three-way tie }},
\end{gathered}
$$

whereas the expected utility of this voter approving of both $i$ and $j$ at event $z$ equals:

$$
\begin{gathered}
U_{t}[i j \mid n \tau]=\overbrace{\mathbb{P}\left[\operatorname{piv}_{i}-i j\right] \cdot 1+\mathbb{P}\left[\text { piv }_{j}-i j\right] \cdot u+\mathbb{P}\left[\operatorname{piv}_{k}-i j\right] \cdot 0}^{\text {Single-winner events }} \\
+\overbrace{\mathbb{P}\left[\operatorname{piv}_{i j}-i j\right]\left(\frac{1+u}{2}\right)+\mathbb{P}\left[\operatorname{piv}_{i k}-i j\right]\left(\frac{1}{2}\right)+\mathbb{P}\left[\operatorname{piv}_{j k}-i j\right]\left(\frac{u}{2}\right)}^{\text {Two-way pivots }} \\
+\overbrace{\mathbb{P}\left[\operatorname{piv}_{a b c}-i j\right]\left(\frac{1+u}{3}\right)}^{\text {Three-way tie }},
\end{gathered}
$$

Thus, the difference between both expected utilities, $U_{t}[i \mid n \tau]-U_{t}[i, j \mid n \tau]$ equals:

$$
\begin{aligned}
& \left(\mathbb{P}\left[\operatorname{piv}_{i}-i\right]+\mathbb{P}\left[\operatorname{piv}_{j}-i\right] u+\mathbb{P}\left[\operatorname{piv}_{i j}-i\right] \frac{1+u}{2}+\mathbb{P}\left[\operatorname{piv}_{i k}-i\right] \frac{1}{2}+\mathbb{P}\left[\operatorname{piv}_{j k}-i\right] \frac{u}{2}+\mathbb{P}\left[\operatorname{piv}_{a b c}-i\right] \frac{1+u}{3}\right)- \\
& \left(\mathbb{P}\left[\operatorname{piv}_{i}-i j\right]+\mathbb{P}\left[\operatorname{piv}_{j}-i j\right] u+\mathbb{P}\left[\operatorname{piv}_{i j}-i j\right] \frac{1+u}{2}+\mathbb{P}\left[\operatorname{piv}_{i k}-i j\right] \frac{1}{2}+\mathbb{P}\left[\operatorname{piv}_{j k}-i j\right] \frac{u}{2}+\mathbb{P}\left[\operatorname{piv}_{a b c}-i j\right] \frac{1+u}{3}\right) .
\end{aligned}
$$

The previous expression can be simplified due to the following observations.

Note first that $\left(\operatorname{piv}_{i}-i\right)=\left(\operatorname{piv}_{i}-i j\right) \cup\left(\operatorname{piv}_{i j}-i j\right)$. Hence,

$$
\mathbb{P}\left[\operatorname{piv}_{i}-i\right]-\mathbb{P}\left[\operatorname{piv}_{i}-i j\right]-\mathbb{P}\left[\operatorname{piv}_{i j}-i j\right] \frac{1+u}{2}=\mathbb{P}\left[\operatorname{piv}_{i j}-i j\right] \frac{1-u}{2}
$$


Similarly, observe that $\left(\operatorname{piv}_{j}-i j\right)=\left(\operatorname{piv}_{j}-i\right) \cup\left(\operatorname{piv}_{i j}-i\right) \cup\left(\operatorname{piv}_{j k}-i\right) \cup\left(\operatorname{piv}_{a b c}-i\right)$. Thus,

$$
\begin{aligned}
& \mathbb{P}\left[\operatorname{piv}_{j}-i\right] u+\mathbb{P}\left[\operatorname{piv}_{i j}-i\right] \frac{1+u}{2}+\mathbb{P}\left[\operatorname{piv}_{j k}-i\right] \frac{u}{2}+\mathbb{P}\left[\operatorname{piv}_{a b c}-i\right] \frac{1+u}{3}-\mathbb{P}\left[\operatorname{piv}_{j}-i j\right] u \\
& =\mathbb{P}\left[\operatorname{piv}_{j}-i\right](u-u)+\mathbb{P}\left[\operatorname{piv}_{i j}-i\right]\left(\frac{1+u}{2}-u\right)+\mathbb{P}\left[\operatorname{piv}_{j k}-i\right]\left(\frac{u}{2}-u\right)+\mathbb{P}\left[\operatorname{piv}_{a b c}-i\right]\left(\frac{1+u}{3}-u\right) \\
& =\mathbb{P}\left[\operatorname{piv}_{i j}-i\right] \frac{1-u}{2}+\mathbb{P}\left[\operatorname{piv}_{j k}-i\right]\left(-\frac{u}{2}\right)+\mathbb{P}\left[\operatorname{piv}_{a b c}-i\right] \frac{1-2 u}{3}
\end{aligned}
$$

Finally, following a similar reasoning, one can see that $\left(\operatorname{piv}_{i k}-i\right)=\left(\operatorname{piv}_{i k}-i j\right) \cup\left(\operatorname{piv}_{a b c}-i j\right)$ and therefore,

$$
\begin{aligned}
\mathbb{P}\left[\operatorname{piv}_{i k}-i\right] \frac{1}{2}-\mathbb{P}\left[\operatorname{piv}_{i k}-i j\right] \frac{1}{2}-\mathbb{P}\left[\operatorname{piv}_{a b c}-i j\right] \frac{1+u}{3} & =\mathbb{P}\left[\operatorname{piv}_{a b c}-i j\right]\left(\frac{1}{2}-\frac{1+u}{3}\right) \\
& =\mathbb{P}\left[\operatorname{piv}_{a b c}-i j\right] \frac{1-2 u}{6}
\end{aligned}
$$

Combining equalities (5) to (7), (1) can be rewritten as :

$$
\begin{aligned}
\left(\mathbb{P}\left[\operatorname{piv}_{i j}-i\right]+\mathbb{P}\left[\operatorname{piv}_{i j}-i j\right]\right) \frac{1-u}{2} & +\left(\mathbb{P}\left[\operatorname{piv}_{j k}-i\right]+\mathbb{P}\left[\operatorname{piv}_{j k}-i j\right]\right)\left(-\frac{u}{2}\right) \\
& +\mathbb{P}\left[\operatorname{piv}_{a b c}-i\right] \frac{1-2 u}{3}+\mathbb{P}\left[\operatorname{piv}_{a b c}-i j\right] \frac{1-2 u}{6} .
\end{aligned}
$$

Finally, we obtain the desired expression by observing that $\operatorname{piv}_{i j}^{t}=\left(\operatorname{piv}_{i j}-i\right) \cup\left(\operatorname{piv}_{i j}-i j\right)$, $\operatorname{piv}_{j k}^{t}=\left(\operatorname{piv}_{j k}-i\right) \cup\left(\operatorname{piv}_{j k}-i j\right), \operatorname{tie}_{a b c}^{t, 1}=\left(\operatorname{piv}_{a b c}-i\right)$ and $\operatorname{tie}_{a b c}^{t, 2}=\left(\operatorname{piv}_{a b c}-i j\right)$.

\section{A.2. Proof of Theorem 1}

Proof. As a direct application of the Magnitude Equivalence Theorem in Núñez [2010], one can show that for any ballot profile $\tau$, any type $t$ and any pair $i j$ of candidates we have:

$$
\mu\left[\operatorname{piv}_{i j}^{t}\right]=\mu\left\{s_{i}=s_{j} \geq s_{k}\right\} \quad \text { and } \quad \mu\left[\operatorname{tie}_{a b c}^{t, 1}\right]=\mu\left[\operatorname{tie}_{a b c}^{t, 2}\right]=\mu\left[\operatorname{tie}_{a b c}\right] .
$$

It remains to be shown that for any ballot profile $\tau, \mu\left\{s_{i}=s_{j} \geq s_{k}\right\} \in\left\{\mu\left[\operatorname{tie}_{i j}\right], \mu\left[\operatorname{tie}_{a b c}\right]\right\}$. To do so, we first apply the Dual Magnitude Theorem of Myerson [2002]. We can write:

$$
\mu\left[\operatorname{tie}_{a b c}\right]=\min _{x, y \in \mathbb{R}} f(x, y), \quad \mu\left\{s_{i}=s_{j} \geq s_{k}\right\}=\min _{x \in \mathbb{R}, y \geq 0} f(x, y), \quad \mu\left[\operatorname{tie}_{i j}\right]=\min _{x \in \mathbb{R}, y=0} f(x, y)
$$

where

$$
f(x, y)=\tau_{i} e^{x+y}+\tau_{i k} e^{x}+\tau_{j} e^{-x}+\tau_{j k} e^{-(x+y)}+\tau_{k} e^{-y}+\tau_{i j} e^{y}-1 .
$$

The function $f$ is strictly convex as $\frac{\partial^{2} f}{\partial x^{2}}=e^{x}\left(\tau_{i} e^{y}+\tau_{i k}\right)+e^{-x}\left(\tau_{j}+\tau_{j k} e^{-y}\right)>0$ and:

$$
\begin{aligned}
\frac{\partial^{2} f}{\partial x^{2}} \times \frac{\partial^{2} f}{\partial y^{2}}-\left(\frac{\partial^{2} f}{\partial x \partial y}\right)^{2}= & {\left[e^{x}\left(\tau_{i} e^{y}+\tau_{i k}\right)+e^{-x}\left(\tau_{j}+\tau_{j k} e^{-y}\right)\right] \times } \\
& {\left[e^{y}\left(\tau_{i} e^{x}+\tau_{i j}\right)+e^{-y}\left(\tau_{k}+\tau_{j k} e^{-x}\right)\right]-\left(\tau_{i} e^{x+y}+\tau_{j k} e^{-(x+y)}\right)^{2}>0 . }
\end{aligned}
$$

As $|f(x, y)|$ becomes large when either $|x|$ or $|y|$ is large, $f$ admits a unique critical point $\left(x^{* *}, y^{* *}\right) \in \mathbb{R}^{2}$. The value $y^{* *}$ solves for $\min _{y \in \mathbb{R}} f\left(x^{*}(y), y\right)$, where $x^{*}(y)=\arg \min _{x \in \mathbb{R}} f(x, y)$ is uniquely defined. We have two cases to consider: 
- Either $\frac{\partial f}{\partial y}\left(x^{*}(0), 0\right)>0$. In that case, $y^{* *}<0$, and we obtain that $\mu\left\{s_{i}=s_{j} \geq s_{k}\right\}, \mu\left[\operatorname{tie}_{i j}\right]>$ $\mu\left[\right.$ tie $\left._{a b c}\right]$. Moreover, we have:

$$
\mu_{i j}=\min _{x \in \mathbb{R}, y \geq 0} f(x, y)=\min _{y \geq 0} f\left(x^{*}(y), y\right)=f\left(x^{*}(0), 0\right)=\min _{x \in \mathbb{R}, y=0} f(x, y)=\mu\left[\text { tie }_{i j}\right] .
$$

- Or $\frac{\partial f}{\partial y}\left(x^{*}(0), 0\right) \leq 0$. In that case, $y^{* *} \geq 0$, and we obtain that $\mu\left\{s_{i}=s_{j} \geq s_{k}\right\}=\mu\left[\right.$ tie $\left._{a b c}\right]$.

To conclude, the value $x^{*}(0)$ is obtained by setting

$$
0=\frac{\partial f}{\partial x}\left(x^{*}(0), 0\right)=e^{x^{*}(0)}\left(\tau_{i}+\tau_{i k}\right)-e^{-x^{*}(0)}\left(\tau_{j}+\tau_{j k}\right),
$$

and we get $e^{x^{*}(0)}=\sqrt{\frac{\tau_{j}+\tau_{j k}}{\tau_{i}+\tau_{i k}}}$. Finally, we write

$$
\frac{\partial f}{\partial y}\left(x^{*}(0), 0\right)=\tau_{i} e^{x^{*}(0)}+\tau_{i j}-\tau_{k}-\tau_{j k} e^{-x^{*}(0)}=\delta_{i j}(\tau) .
$$

As wanted, we have shown that $\mu\left\{s_{i}=s_{j} \geq s_{k}\right\}$ is equal to either $\mu\left[\operatorname{tie}_{i j}\right]$, if $\delta_{i j}(\tau)>0$, or to $\mu\left[\right.$ tie $\left._{a b c}\right]$ otherwise.

\section{A.3. Proof of Proposition 2}

Proof. By application of the Dual Magnitude Theorem, as in the proof of Theorem 1, we have

$$
\mu_{a b c}=\min _{x, y \in \mathbb{R}} \tau_{a} e^{x+y}+\tau_{a c} e^{x}+\tau_{b} e^{-x}+\tau_{b c} e^{-(x+y)}-1,
$$

which implies that $\mu_{a b c}=-\left(\sqrt{\tau_{a}}-\sqrt{\tau_{b c}}\right)^{2}-\left(\sqrt{\tau_{b}}-\sqrt{\tau_{a c}}\right)^{2}$.

By application of Theorem 1, the formula for the magnitude $\mu_{a b}$ depends on the sign of:

$$
\delta_{a b}(\tau)=\tau_{a} \sqrt{\frac{\tau_{b}+\tau_{b c}}{\tau_{a}+\tau_{a c}}}-\tau_{b c} \sqrt{\frac{\tau_{a}+\tau_{a c}}{\tau_{b}+\tau_{b c}}} .
$$

We obtain that

$$
\delta_{a b}(\tau)>0 \Leftrightarrow \tau_{a}\left(\tau_{b}+\tau_{b c}\right)>\tau_{b c}\left(\tau_{a}+\tau_{a c}\right) \quad \Leftrightarrow \quad \tau_{a} \tau_{b}>\tau_{a c} \tau_{b c} .
$$

When this inequality is satisfied, we may write:

$$
\mu_{a b}=\mu\left[\operatorname{tie}_{a b}\right]=\min _{x \in \mathbb{R}, y=0} \tau_{a} e^{x+y}+\tau_{a c} e^{x}+\tau_{b} e^{-x}+\tau_{b c} e^{-(x+y)}-1=-\left(\sqrt{\tau_{a}+\tau_{a c}}-\sqrt{\tau_{b}+\tau_{b c}}\right)^{2} .
$$

The formulas for the magnitudes $\mu_{a c}$ and $\mu_{b c}$ are obtained in a similar fashion and are hence omitted.

\section{A.4. Proof of Theorem 2}

We first prove a lemma, relating a candidates' expected scores to pivot magnitudes.

Lemma 1. Let $\sigma$ be a strategy profile with associated expected score $\gamma$. We have the following:

(i) If $\gamma_{a}, \gamma_{b}>\gamma_{c}$, then $\mu_{a b}>\mu_{a b c}$

(ii) If $\gamma_{a}=\gamma_{b}>\gamma_{c}$, then $\mu_{a b}>\mu_{a c}=\mu_{b c}=\mu_{a b c}$

(iii) If $\gamma_{a}>\gamma_{b}, \gamma_{c}$, then $\mu_{b c}=\mu_{a b c}$ 
(iv) If $\gamma_{a}>\gamma_{b}=\gamma_{c}$, then $\mu_{b c}=\mu_{a b c}$ and $\left(\mu_{a b}>\mu_{a b c}\right.$ or $\left.\tau_{b}=\tau_{a c}=0\right)$ and $\left(\mu_{a c}>\mu_{a b c}\right.$ or $\left.\tau_{c}=\tau_{a b}=0\right)$

Proof. The proof relies on Theorem 1. For (i), let us assume that $\gamma_{b} \geq \gamma_{a}$, so that we have $\tau_{b}+\tau_{b c} \geq \tau_{a}+\tau_{a c}$. We write:

$$
\delta_{a b}(\tau)=\tau_{a} \sqrt{\frac{\tau_{b}+\tau_{b c}}{\tau_{a}+\tau_{a c}}}+\tau_{a b}-\tau_{b c} \sqrt{\frac{\tau_{a}+\tau_{a c}}{\tau_{b}+\tau_{b c}}}-\tau_{c} \geq \tau_{a}+\tau_{a b}-\tau_{b c}-\tau_{c}=\gamma_{a}-\gamma_{c}>0
$$

Hence, $\mu_{a b}>\mu_{a b c}$, as desired.

For (ii), it suffices to show that $\mu_{a c}=\mu_{a b c}$. As $\gamma_{c} \leq \gamma_{a}$, we obtain as before $\delta_{a c}(\tau) \leq \gamma_{a}-\gamma_{b}=0$. Hence, $\mu_{a c}=\mu_{a b c}$, as desired.

For (iii), we use the fact that $\gamma_{c} \leq \gamma_{b}$. We obtain $\delta_{b c}(\tau) \leq \gamma_{b}-\gamma_{a}<0$. Hence, $\mu_{b c}=\mu_{a b c}$, as desired.

For (iv), it will be easier to show the claim with the ordering $\gamma_{b}>\gamma_{a}=\gamma_{c} \cdot{ }^{14}$ As $\gamma_{b}>\gamma_{a}$, we obtain $\delta_{a b}(\tau) \geq \gamma_{a}-\gamma_{c}=0$, with a strict inequality only if $\tau_{a}>0$ or $\tau_{b c}>0$. We thus have two possibilities: either $\mu_{a b}>\mu_{a b c}$ (if $\delta_{a b}(\tau)>0$ ) or $\tau_{a}=\tau_{b c}=0$. The argument for $\mu_{a c}$ is the same.

We now prove Theorem 2.

Proof. We now consider each of the two possible cases of non-discriminatory equilibrium.

Equilibrium with: $\mu_{a b}=\mu_{a c}>\mu_{b c}$.

Assume that $\sigma$ is an equilibrium with $\mu_{a b}=\mu_{a c}>\mu_{b c}$. As $\mu_{a b}, \mu_{a c}>\mu_{b c}$, we get from Theorem 1 that

$$
\mu_{a b}=-\left(\sqrt{\tau_{a}+\tau_{a c}}-\sqrt{\tau_{b}+\tau_{b c}}\right)^{2}, \quad \mu_{a c}=-\left(\sqrt{\tau_{a}+\tau_{a b}}-\sqrt{\tau_{c}+\tau_{b c}}\right)^{2}
$$

and that these formulas remain valid in a neighborhood of $\tau$. Therefore, there is a ballot profile $\tau^{\prime}$ arbitrarily close to $\tau$ such that $\mu_{a b}>\mu_{a c}>\mu_{b c}$, and a ballot profile $\tau^{\prime \prime}$ for which $\mu_{a c}>\mu_{a b}>\mu_{b c}$. If $\sigma$ was stable, $\sigma_{t}$ would be a best response to both $\tau^{\prime}$ and $\tau^{\prime \prime}$. We obtain a contradiction by considering type $t=c a$ for which $\operatorname{BR}\left(c a \mid n \tau^{\prime}\right)=a c$ and $\operatorname{BR}\left(c a \mid n \tau^{\prime \prime}\right)=c$ for $n$ large enough.

Equilibrium with : $\mu_{a b}=\mu_{a c}=\mu_{b c}$.

Assume that $\sigma$ is an equilibrium with $\mu_{a b}=\mu_{a c}=\mu_{b c}$. By application of Lemma 1, we have two possibilities: either $\gamma_{a}=\gamma_{b}=\gamma_{c}$ or $\left(\gamma_{a}>\gamma_{b}=\gamma_{c}\right.$ and $\left.\tau_{b}=\tau_{a c}=\tau_{c}=\tau_{a b}=0\right)$. The second case can be ruled out: we have by assumption $r_{b a}>0$, so that $\tau_{b}>0$ or $\tau_{a b}>0$.

We can find $\tau^{\prime}$ and $\tau^{\prime \prime}$ arbitrarily close to $\tau$ such that $\gamma_{a}^{\prime}=\gamma_{b}^{\prime}>\gamma_{c}^{\prime}$ and $\gamma_{a}^{\prime \prime}=\gamma_{c}^{\prime \prime}>\gamma_{b}^{\prime \prime}$. In the first case, pivot $a b$ dominates, while pivot $a c$ dominates in the second case, by application of (ii) in Lemma 1 . We get a contradiction as before by considering $t=c a$.

\footnotetext{
${ }^{14}$ The discriminant $\delta_{a b}(\tau)$ is defined as the difference between the expected scores of $a$ and $c$ conditional on tie ${ }_{a b}$. One could also prove the claim directly, by defining the symmetric discriminant defined as the difference between the expected scores of $b$ and $c$ conditional on tie $a b$.
} 


\section{A.5. Proof of Theorem 3}

Proof. Let $\sigma$ be a direct equilibrium satisfying $\mu_{a b}>\mu_{a c}>\mu_{b c}$. It follows from condition (*) that $\gamma_{a}=\tau_{a}+\tau_{a c}, \gamma_{b}=\tau_{b}+\tau_{b c}$ and $\gamma_{c}=\tau_{a c}+\tau_{b c}$. We observe that $\tau_{a c} \tau_{b c}<\tau_{a} \tau_{b}$ since we have $\mu_{a b}>\mu_{a b c}$ and $\tau_{b}<\tau_{a c}$ since $\mu_{a c}>\mu_{a b c}$. Combining the pair of previous inequalities implies that $\tau_{b c}<\tau_{a}$, which, in turn, implies that $\gamma_{a}>\gamma_{c}$. Moreover, the inequality $\tau_{b}<\tau_{a c}$ implies on its own that $\gamma_{c}>\gamma_{b}$. It follows that $\gamma_{a}>\gamma_{c}>\gamma_{b}$.

Let $\sigma$ be an indirect equilibrium. By a direct application of Lemma 1, it cannot be that $\gamma_{c}>\gamma_{a}$ (considering all the possible values for $\gamma_{b}$ ). Therefore $\gamma_{a} \geq \gamma_{c}$, and similarly $\gamma_{b} \geq \gamma_{c}$.

\section{A.6. Proof of Proposition 3}

Proof. Consider a preference profile $r^{\prime}$ in a neighborhood of $r$ (the symmetric Condorcet cycle), say for each $t \in \mathscr{T},\left|r_{t}-r_{t}^{\prime}\right| \leq \varepsilon$. We have:

$$
\frac{r_{a}}{r_{-a}} \leq \frac{1 / 3+\varepsilon}{1 / 3-\varepsilon} \quad \text { and } \quad \frac{r_{c a}}{r_{b a}} \geq \frac{1 / 3-\varepsilon}{\varepsilon} .
$$

For $\varepsilon$ small enough, we have that $r_{c a} / r_{b a}>r_{a} / r_{-a}$. Thus, no direct equilibrium exists with $\mu_{a b}>\mu_{a c}>\mu_{b c}$. Moreover, as $r_{c a} / r_{b a}<1$, no direct equilibrium exists with $\mu_{a c}>\mu_{a b}>\mu_{b c}$.

A symmetric argument applies for direct equilibria where $b$ or $c$ belongs to the two first pivots. We conclude that there exists a neighborhood of $r$ for which no direct equilibrium exists. This set of preference profiles is of positive measure, which concludes the proof.

\section{A.7. Proof of Theorem 4}

Proof. Let $\sigma$ be a stable equilibrium with $\mu_{a b}>\mu_{a c}=\mu_{b c}$ and let $t \in\{a c, b c\}$.

1. The proof relies on the offset theorem introduced in Myerson [2000]. The theorem establishes that events differing from each other by a single translation have a probability ratio that can be computed using the offset ratios.

As $\sigma$ is an equilibrium satisfying $\mu_{a b}>\mu_{a c}=\mu_{b c}$, we must have $\tau_{a b}=\tau_{c}=0$ (as $\mu_{a b}$ dominates, $a b$ and $c$ are never best responses), $\tau_{a} \geq \tau_{b c}\left(\operatorname{as} \mu_{b c}=\mu_{a b c}\right)$ and $\tau_{b} \geq \tau_{a c}\left(\right.$ as $\left.\mu_{a c}=\mu_{a b c}\right)$.

Claim: The offset ratios are identical in events $\operatorname{piv}_{a c}, \operatorname{piv}_{b c}$ and tie $a b c$.

Let us prove the claim for $\operatorname{piv}_{a c}$ and tie ${ }_{a b c}$. By slightly abusing notation, we can write $\operatorname{piv}_{a c}=\left\{Z_{a}=Z_{b c}\right\} \cap\left\{Z_{a c}>Z_{b}\right\}$ and tie $a b c=\left\{Z_{a}=Z_{b c}\right\} \cap\left\{Z_{a c}=Z_{b}\right\}$. We first observe that the offset ratios of ballots $a$ and $b c$ will be the same for the two events: they are each equal to the corresponding offset ratio in the event $\left\{Z_{a}=Z_{b c}\right\}$. Moreover, as $\tau_{b} \geq \tau_{a c}$, we have that for any $n$, the most likely outcome in the event $\left\{Z_{a c}>Z_{b}\right\}$ belongs in fact to the event $\left\{Z_{a c}=Z_{b}+1\right\}$, which implies that the two events admit the same offset ratios (from the definition of offset ratios). In turn, the offset ratios of ballots $a c$ and $b$ are the same for events $\left\{Z_{a c}=Z_{b}+1\right\}$ and $\left\{Z_{a c}=Z_{b}\right\}$, which proves the claim.

From now on, we can thus write $\phi_{m}$ for the offset ratio of ballot $m$. We know from the formulas derived in the main text that $\phi_{m}>0$ for any $m \in\{a, b, a c, b c\}$. Applying the offset 
theorem, we obtain that:

$$
\lim _{n \rightarrow \infty} \frac{\mathbb{P}\left[\text { piv }_{a b c}^{t, 1} \mid n \tau\right]}{\mathbb{P}\left[\text { tie }_{a b c} \mid n \tau\right]}=\phi_{t_{1}} \quad \text { and } \quad \lim _{n \rightarrow \infty} \frac{\mathbb{P}\left[\left[\text { piv }_{a b c}^{t, 2} \mid n \tau\right]\right.}{\mathbb{P}\left[\operatorname{tie}_{a b c} \mid n \tau\right]}=\phi_{t_{1} t_{2}}
$$

For the pivots with two candidates, let us focus on pivot $a c$. With the notations in the statement of the theorem, this corresponds to $i=a$ (the pivot under consideration is $a c$ ) and $j=b$ ( $b$ is the candidate not appearing in the pivot). Applying the offset theorem, we obtain:

$$
\lim _{n \rightarrow \infty} \frac{\mathbb{P}\left[\operatorname{piv}_{a c}^{t} \mid n \tau\right]}{\mathbb{P}\left[\operatorname{piv}_{a c} \mid n \tau\right]}=\lim _{n \rightarrow \infty}\left(\frac{\mathbb{P}\left[\operatorname{piv}_{a c}-t_{1} \mid n \tau\right]}{\mathbb{P}\left[\operatorname{piv}_{a c} \mid n \tau\right]}+\frac{\mathbb{P}\left[\operatorname{piv}_{a c}-t_{1} t_{2} \mid n \tau\right]}{\mathbb{P}\left[\operatorname{piv}_{a c} \mid n \tau\right]}\right)=\phi_{t_{1}}+\phi_{t_{1} t_{2}} .
$$

What remains to be done is to compare the likelihood of events $\operatorname{piv}_{a c}$ and tie $a b c$. We rely on two observations: first, $\operatorname{piv}_{a c}=\left(\operatorname{piv}_{a c} \cup \operatorname{tie}_{a b c}\right)-b$, as $\left\{Z_{a c}>Z_{b}\right\}=\left\{Z_{a c} \geq Z_{b}\right\}-b$. Second, the offset ratio of $b$ in $\operatorname{piv}_{a c} \cup$ tie $_{a b c}$ is the same as in any of these two events, precisely because $\phi_{b}$ is the same in the two events. Applying the offset theorem, we can write:

$$
\lim _{n \rightarrow \infty} \frac{\mathbb{P}\left[\operatorname{piv}_{a c} \mid n \tau\right]}{\mathbb{P}\left[\operatorname{piv}_{a c} \mid n \tau\right]+\mathbb{P}\left[\operatorname{tie}_{a b c} \mid n \tau\right]}=\lim _{n \rightarrow \infty} \frac{\mathbb{P}\left[\left(\operatorname{piv}_{a c} \cup \operatorname{tie}_{a b c}\right)-b \mid n \tau\right]}{\mathbb{P}\left[\operatorname{piv}_{a c} \cup \operatorname{tie}_{a b c} \mid n \tau\right]}=\phi_{b}
$$

From this we obtain that $\lim _{n \rightarrow \infty} \frac{\mathbb{P}\left[\operatorname{piv}_{a c} \mid n \tau\right]}{\mathbb{P}\left[\operatorname{tie}_{a b c} \mid n \tau\right]}=\frac{\phi_{b}}{1-\phi_{b}}$, and we conclude that

$$
\lim _{n \rightarrow \infty} \frac{\mathbb{P}\left[\operatorname{piv}_{a c}^{t} \mid n \tau\right]}{\mathbb{P}\left[\text { tie }_{a b c} \mid n \tau\right]}=\frac{\phi_{b}}{1-\phi_{b}}\left(\phi_{t_{1}}+\phi_{t_{1} t_{2}}\right) .
$$

Finally, by symmetry of the problem, we obtain a symmetric formula for the pivot $b c$. Note that we have implicitly assumed in the proof that all offset ratios are different from 1 . One can check that the formulas remain valid if some offset ratios reach the value of 1 , concluding the proof of 1 .

2. Applying the previous result, we obtain $\lim _{n \rightarrow \infty} \Delta(t \mid n \tau) / \mathbb{P}[\operatorname{tie} a b c \mid n \tau]=g\left(u_{t}\right)$ with

$$
g\left(u_{t}\right)=\frac{1 / \phi_{t_{1} t_{2}}}{1-1 / \phi_{t_{1} t_{2}}}\left(\phi_{t_{1}}+\phi_{t_{1} t_{2}}\right)\left(\frac{1-u_{t}}{2}\right)+\frac{\phi_{t_{1}}}{1-\phi_{t_{1}}}\left(\phi_{t_{1}}+\phi_{t_{1} t_{2}}\right)\left(\frac{-u_{t}}{2}\right)+\left(2 \phi_{t_{1}}+\phi_{t_{1} t_{2}}\right)\left(\frac{1-2 u_{t}}{6}\right) .
$$

Thus, for $n$ large, the sign of $\Delta(t \mid n \tau)$ is the same as the sign of $g\left(u_{t}\right)$. The function $g$ is decreasing and takes value 0 for $u_{t}^{*} \in(0,1)$, defined by:

$$
u_{t}^{*}=\frac{\left(\phi_{t_{1}}+\phi_{t_{1} t_{2}}\right)\left(\frac{3}{\phi_{t_{1} t_{2}}-1}+1\right)+\phi_{t_{1}}}{\left(\phi_{t_{1}}+\phi_{t_{1} t_{2}}\right)\left(\frac{3}{\phi_{t_{1} t_{2}}-1}+\frac{3}{1 / \phi_{t_{1}}-1}+2\right)+2 \phi_{t_{1}}} .
$$

As $\sigma$ is an equilibrium, we conclude using Proposition 1 that $u_{t}<u_{t}^{*} \Rightarrow \sigma_{t}=t_{1}$ and $u_{t}>u_{t}^{*} \Rightarrow$ $\sigma_{t}=t_{1} t_{2}$.

Note again that, while we have assumed that the offset ratios are different from 1 , the result remains valid (defining $u_{t}^{*}$ as a limit), when some offset ratios reach this value.

\section{Appendix B. Alternative model With Correlation NeGleCt}

In the benchmark model, voters condition on the ballot profile $\tau$ to determine their best response (that is, they compute the sign of $\Delta(t \mid n \tau)$ for each type $t$ ). If voters neglect this correlation, they compute pivot probabilities assuming that the scores of the different candidates 
are independent random variables. In other words, rather that conditioning on the ballot profile $\tau$ to compute pivot probabilities, a voter conditions on the score distribution $\gamma$ assuming that each pair $S_{i}, S_{j}$ is a pair of independent random variables with respective expected scores $\gamma_{i}$ and $\gamma_{j}$. As a simple corollary of Theorem 1, one can write that for any score distribution $\gamma$, any type $t \in \mathscr{T}$ and any pair $a b$ of candidates:

1. $\mu\left[\mathrm{tie}_{a b}\right]=-\left(\sqrt{\gamma_{a}}-\sqrt{\gamma_{b}}\right)^{2}$,

2. $\mu\left[\right.$ tie $\left._{a b c}\right]=3\left(\gamma_{a} \gamma_{b} \gamma_{c}\right)^{\frac{1}{3}}-\gamma_{a}-\gamma_{b}-\gamma_{c}$,

3. $\mu\left[\operatorname{piv}_{a b}^{t}\right]= \begin{cases}\mu\left[\mathrm{tie}_{a b}\right] & \text { if } \gamma_{c}^{2}<\gamma_{a} \gamma_{b}, \\ \mu\left[\mathrm{tie}_{a b c}\right] & \text { if } \gamma_{c}^{2} \leq \gamma_{a} \gamma_{b},\end{cases}$

4. $\mu\left[\mathrm{tie}_{a b c}^{t, 1}\right]=\mu\left[\mathrm{tie}_{a b c}^{t, 2}\right]=\mu\left[\mathrm{tie}_{a b c}\right]$.

Following the methods developed in the article, we obtain Theorem 5.

\section{ApPendix C. TECHNiCAL Discussion}

In this section, we provide further justifications for our focus on stable equilibria. Starting from a weaker definition of a (large) equilibrium, we first show that discriminatory and stable equilibria coincide. Then, we prove that, for generic preference profiles, any equilibrium is stable. Finally, we demonstrate that discriminatory equilibria, as defined by Myerson (with a condition on relative pivot probabilities), coincide with the stricter definition of discriminatory equilibria we use (with a condition on pivot magnitudes).

We start by stating a definition of a large equilibrium, considering the limit behaviour when $n \rightarrow \infty$.

Definition 3. The pure strategy profile $\sigma=\left(\sigma_{t}\right)_{t \in T}$ is a large equilibrium if there is some $n_{0}$ such that for any $n \geq n_{0}$, for each $t \in T, \sigma_{t} \in B R(t \mid n \tau)$.

This definition is more practical, even if formally more restrictive, than that of a large equilibrium in Myerson [2000], which is based the convergence of a sequence of finite- $n$-equilibria.

\section{C.1. Equivalence between stable and discriminatory equilibria}

We first provide a result reciprocal to Theorem 2: any discriminatory large equilibrium is stable.

Proposition 5. Let $\sigma$ be a discriminatory large equilibrium $\left(\mu_{a b}>\mu_{a c}, \mu_{b c}\right)$. Then, $\sigma$ is a stable equilibrium.

Proof. There are two kinds of discriminatory large equilibria: $\operatorname{direct}\left(\mu_{a b}>\mu_{a c}>\mu_{b c}\right)$ and indirect ones $\left(\mu_{a b}>\mu_{a c}=\mu_{b c}\right)$.

For a direct equilibrium, the conditions (*) and (**) identified in Section 4 must be satisfied. These two conditions are strict inequalities on the ballot profile $\tau$. Then, in any neighborhood of $\tau$, the magnitude ordering remains, and the best responses are identical: the equilibrium is indeed stable.

For an indirect equilibrium with $\mu_{a b}>\mu_{a c}=\mu_{b c}$, the conditions of Theorem 4 must be satisfied. As the profile is in pure strategies, and $r$ is assumed to exhibit no tie, we have that (i) all the offset ratios lie strictly between 0 and 1 , so that $u_{a c}^{*}, u_{b c}^{*} \in(0,1)$; and (ii) the inequalities 
on $\tau$ justifying the magnitude ordering are strict. Therefore, in a neighborhood of $\tau$, the magnitude ordering is unchanged, and the values $u_{a c}^{*}, u_{b c}^{*}$ move continuously. It follows that the equilibrium is indeed stable.

\section{C.2. On the generic non-existence of non-stable equilibria}

We establish that pure-strategy equilibria that are not stable only exist for a 0 -measure set of ordinal preference profiles.

Proposition 6. The set of preference profiles $r$ for which a non-stable large equilibrium exists $\left(\mu_{a b}=\mu_{a c}>\mu_{b c}\right.$ or $\left.\mu_{a b}=\mu_{a c}=\mu_{b c}\right)$ is of measure 0 .

Proof. We consider each of the two possible cases of non-discriminatory large equilibrium.

Assume that $\sigma$ is an equilibrium with $\mu_{a b}=\mu_{a c}>\mu_{b c}$. As $\mu_{a b}, \mu_{a c}>\mu_{b c}$, we get from Theorem 1 that

$$
\mu_{a b}=-\left(\sqrt{\tau_{a}+\tau_{a c}}-\sqrt{\tau_{b}+\tau_{b c}}\right)^{2}=\mu_{a c}=-\left(\sqrt{\tau_{a}+\tau_{a b}}-\sqrt{\tau_{c}+\tau_{b c}}\right)^{2}
$$

and that these formulas remain valid in a neighborhood of $\tau$. Therefore, such an equilibrium cannot be found generically in a neighborhood of $r$ (as strategies are pure).

Assume that $\sigma$ is an equilibrium with $\mu_{a b}=\mu_{a c}=\mu_{b c}$. As shown in the proof of Theorem 2, the expected scores of two candidates must be equal. This cannot remain true generically in a neighborhood of $r$.

\section{C.3. On the notion of a discriminatory equilibrium}

Myerson [2002] introduces the notion of discriminatory equilibrium in which one pivot event between some pair of candidates is infinitely more likely than any other pivots event. For any pair of candidates $i j$, the event $\operatorname{piv}_{i j}$ is serious if $\lim _{n \rightarrow \infty} \frac{\mathbb{P}_{\left[\operatorname{piv}_{i j} \mid n \tau\right]}}{\sum_{K \subseteq \mathcal{K}} \mathbb{P}\left[\operatorname{piv}_{K} \mid n \tau\right]}>0$. That is, the pivot between $i$ and $j$ is serious if, in the event that a close race exists in the election, the conditional probability that $i$ and $j$ are in this close race has a positive limit as the expected population gets large. A candidate $i$ is said to be serious if there is some other candidate $j$ such that $\operatorname{piv}_{i j}$ is serious. According to Myerson [2002], a large equilibrium is discriminatory if and only if there is a candidate in $\mathscr{K}$ who is not serious whereas we have assumed that a discriminatory equilibrium is characterized by the ordering of the magnitudes being $\mu_{a b}>\mu_{a c}, \mu_{b c}$ up to a relabeling of the candidates. The next theorem shows that both notions are identical.

Theorem 6. If a large equilibrium is not discriminatory: $\mu_{a b}=\mu_{a c} \geq \mu_{a b c}$, then the relative probabilities of pivots $a b$ and ac do not diverge: $\mathbb{P}\left[\operatorname{piv}_{a b} \mid n \tau\right] / \mathbb{P}\left[\operatorname{piv}_{a c} \mid n \tau\right]$ is bounded above and bounded away from 0.

Proof. Let $\sigma$ be a discriminatory equilibrium with $\mu_{a b}=\mu_{a c}=\mu_{b c}$ and assume that pivot $a b$ is infinitely more likely than the other pivots. We can apply the formula in Proposition 2 for the magnitudes. The equality of all magnitudes imply that $\tau_{a}=\tau_{b c}$ and $\tau_{b}=\tau_{a c}$. Without loss of generality, we may assume that $\tau_{a} \geq \tau_{b}$. Let us show that, in this case, pivot $a c$ is no less likely than pivot $a b$. While the argument is done for the events piv $_{K}$ it extends to the events piv ${ }_{K}^{t}$. 
We have $Z_{a}, Z_{b c} \sim \mathscr{P}\left(\tau_{a} n\right)$ and $Z_{b}, Z_{a c} \sim \mathscr{P}\left(\tau_{b} n\right)$. We write:

$$
\begin{aligned}
\mathbb{P}\left[\operatorname{piv}_{a b} \mid n \tau\right] & =\mathbb{P}\left[Z_{a}+Z_{a c}=Z_{b}+Z_{b c}>Z_{a c}+Z_{b c} \mid n \tau\right] \\
& =\mathbb{P}\left[Z_{a}+Z_{a c}=Z_{b}+Z_{b c} \mid n \tau\right] \mathbb{P}\left[Z_{b}>Z_{a c}\left|Z_{b}-Z_{a c}=Z_{a}-Z_{b c}\right| n \tau\right] \\
& =\frac{1}{2} \mathbb{P}\left[Z_{a}+Z_{a c}=Z_{b}+Z_{b c} \mid n \tau\right] \text { and } \\
\mathbb{P}\left[\operatorname{piv}_{a c} \mid n \tau\right] & =\mathbb{P}\left[Z_{a}+Z_{a c}=Z_{a c}+Z_{b c}>Z_{b}+Z_{b c} \mid n \tau\right] \\
& =\mathbb{P}\left[Z_{a}=Z_{b c} \mid n \tau\right] \mathbb{P}\left[Z_{a c}>Z_{b} \mid n \tau\right] \\
& =\frac{1}{2} \mathbb{P}\left[Z_{a}=Z_{b c} \mid n \tau\right] \\
& \geq \mathbb{P}\left[\operatorname{piv}_{a b} \mid n \tau\right] .
\end{aligned}
$$

We obtain the desired contradiction: pivot $a b$ cannot be infinitely more likely than pivot $a c$ at the limit.

Finally, let us consider an equilibrium $\sigma$ with $\mu_{a b}=\mu_{a c}>\mu_{b c}$ and assume that pivot $a b$ is infinitely more likely than the other pivots. Applying the formula in Proposition 2, we must have:

$$
\left\{\begin{array}{l}
\tau_{a c} \tau_{b c}<\tau_{a} \tau_{b}, \\
\tau_{b}<\tau_{a c}, \\
\mu_{a b}=2 \sqrt{\left(\tau_{a}+\tau_{a c}\right)\left(\tau_{b}+\tau_{b c}\right)}-1=2 \sqrt{\tau_{a} \tau_{b c}}+\tau_{b}+\tau_{a c}-1=\mu_{a c} .
\end{array}\right.
$$

We can show that: $\mathbb{P}\left[\operatorname{piv}_{a c} \mid n \tau\right]=\mathbb{P}\left[Z_{a}=Z_{b c}, Z_{a c}>Z_{b} \mid n \tau\right] \sim_{n \rightarrow+\infty} \mathbb{P}\left[Z_{a}=Z_{b c} \mid n \tau\right]$ and we have $\mathbb{P}\left[\operatorname{piv}_{a b} \mid n \tau\right]=\mathbb{P}\left[Z_{a}+Z_{a c}=Z_{b}+Z_{b c}, Z_{a}>Z_{b c}\right] \leq \mathbb{P}\left[Z_{a}+Z_{a c}=Z_{b}+Z_{b c} \mid n \tau\right]$.

Now, we use the fact that the difference between two Poisson distributions, $Y \sim \mathscr{P}(\lambda)$ and $Y^{\prime} \sim \mathscr{P}\left(\lambda^{\prime}\right)$, follows a Skellam distribution, and in particular:

$$
\mathbb{P}\left(Y=Y^{\prime}\right)=e^{-\left(\lambda+\lambda^{\prime}\right)} I_{0}\left(2 \sqrt{\lambda \lambda^{\prime}}\right),
$$

where $I_{0}$ is the modified Bessel function of the first kind. This function admits the following asymptotic development for $y$ large:

$$
I_{0}(y) \sim_{y \rightarrow \infty} \frac{e^{y}}{\sqrt{2 \pi y}}, \quad \text { which yields } \quad \ln \left(I_{0}(y)\right)=y-\frac{1}{2} \ln (2 \pi y)+o_{y \rightarrow \infty}(1) .
$$

Using this development, we obtain:

$$
\begin{aligned}
\frac{\ln \left(\mathbb{P}\left[Z_{a}=Z_{b c} \mid n \tau\right]\right)}{n} & =\left(-\tau_{a}-\tau_{b c}+2 \sqrt{\tau_{a} \tau_{b c}}\right)-\frac{1}{2 n} \ln \left(4 \pi n \sqrt{\tau_{a} \tau_{b c}}\right)+o_{n \rightarrow \infty}(1) \\
& =\mu_{a c}-\frac{1}{2 n} \ln \left(4 \pi n \sqrt{\tau_{a} \tau_{b c}}\right)+o_{n \rightarrow \infty}(1)
\end{aligned}
$$


and

$$
\begin{aligned}
\frac{\ln \left(\mathbb{P}\left[Z_{a}+Z_{a c}=Z_{b}+Z_{b c} \mid n \tau\right]\right)}{n}= & \left(-1+2 \sqrt{\left(\tau_{a}+\tau_{a c}\right)\left(\tau_{b}+\tau_{b c}\right)}\right) \\
& -\frac{1}{2 n} \ln \left(4 \pi n \sqrt{\left(\tau_{a}+\tau_{a c}\right)\left(\tau_{b}+\tau_{b c}\right)}\right)+o_{n \rightarrow \infty}(1) \\
= & \mu_{a b}-\frac{1}{2 n} \ln \left(4 \pi n \sqrt{\left(\tau_{a}+\tau_{a c}\right)\left(\tau_{b}+\tau_{b c}\right)}\right)+o_{n \rightarrow \infty}(1) .
\end{aligned}
$$

As we have assumed $\mu_{a b}=\mu_{a c}$, we obtain:

$$
\frac{\mathbb{P}\left[\operatorname{piv}_{a c} \mid n \tau\right]}{\mathbb{P}\left[\operatorname{piv}_{a b} \mid n \tau\right]} \geq \frac{\sqrt{\left(\tau_{a}+\tau_{a c}\right)\left(\tau_{b}+\tau_{b c}\right)}}{\sqrt{\tau_{a} \tau_{b c}}}+o_{n \rightarrow \infty}(1) .
$$

This contradicts the assumption that pivot $a b$ becomes infinitely more likely than the other pivots at the limit. 